\title{
Threshold Boolean Form for Joint Probabilistic Constraints with Random Technology Matrix
}

\author{
Alexander Kogan, Miguel A. Lejeune ${ }^{\dagger}$
}

\begin{abstract}
We develop a new modeling and exact solution method for stochastic programming problems that include a joint probabilistic constraint in which the multi-row random technology matrix is discretely distributed. We binarize the probability distribution of the random variables in such a way that we can extract a threshold partially defined Boolean function (pdBf) representing the probabilistic constraint. We then construct a tight threshold Boolean minorant for the pdBf. Any separating structure of the tight threshold Boolean minorant defines sufficient conditions for the satisfaction of the probabilistic constraint and takes the form of a system of linear constraints. We use the separating structure to derive three new deterministic formulations equivalent to the studied stochastic problem. We derive a set of strengthening valid inequalities for the reformulated problems. A crucial feature of the new integer formulations is that the number of integer variables does not depend on the number of scenarios used to represent uncertainty. The computational study, based on instances of the stochastic capital rationing problem, shows that the MIP reformulations are much easier and orders of magnitude faster to solve than the MINLP formulation. The method integrating the derived valid inequalities in a branch-and-bound algorithm has the best performance.
\end{abstract}

Keywords: Stochastic Programming, Boolean Function, Joint Probabilistic Constraint, Random Technology Matrix, Minorant, Threshold Function

\section{Introduction}

Consider the probabilistically constrained mathematical programming problem SRTM with multi-row random technology matrix

$$
\begin{array}{lc}
\text { SRTM : } & q^{\top} x \\
\text { subject to } & A x \geq b \\
& \mathbb{P}(T x \leq d) \geq p \\
& x \in \mathbb{R}_{+}^{|J|}
\end{array}
$$

where $T$ is an $[r \times|J|]$-matrix and its rows $T_{1}^{\top}, \ldots, T_{r}^{\top}$ are discretely distributed random vectors not necessarily independent. The notation $|J|$ refers to the cardinality of the set $J$. Each component $t_{i j}$ of $T_{i}$ is given by $t_{i j}=s_{i j} \xi_{j}$, where $s_{i j} \in \mathbb{R}_{+}^{1}$ is a fixed positive number and $\xi$ is a $|J|$-dimensional random vector. We denote by $x \in \mathbb{R}^{|J|}$ the vector of decision variables, by $p$ a prescribed reliability level, by $\mathbb{P}$ a probability measure, and by $q^{\top} x: \mathbb{R}^{|J|} \rightarrow \mathbb{R}$ the objective function. The system of inequalities (2), with $A \in \mathbb{R}^{e \times|J|}$ and $b \in \mathbb{R}^{e}$, represents the set of deterministic constraints. The constraint (3) is a multirow probabilistic constraint with random technology matrix that ensures that the $r$ inequalities $T_{i}^{\top} x \leq d_{i}$

${ }^{*}$ Rutgers University, Newark, NJ, USA; kogan@rutgers . edu

†George Washington University, Washington, DC, USA; mlejeune@gwu .edu. 
( $i=1, \ldots, r$ ) hold jointly with a probability at least equal to $p$. Without loss of generality, we assume that the components of the $r$-dimensional vector $d$ are constant. If $d$ is random, we can simply introduce a new variable $x_{|J|+1}$ set equal to 1 and look at $\mathbb{P}\left(T x-d x_{|J|+1} \leq 0\right)$. For discretely distributed variables, the feasible set defined by SRTM is non-convex even when all the decision variables are continuous.

Probabilistically constrained problems with random technology matrix have first been studied by Kataoka [15] and van de Panne and Popp [34]. In their pioneering work, these authors consider a onerow probabilistic constraint with random technology matrix

$$
\mathbb{P}\left(\xi^{\top} x \leq d\right) \geq p
$$

where $\xi$ has normal distribution with $E[\xi]=\mu$ and variance-covariance matrix $\Sigma$. They show that (5) is equivalent to the constraint

$$
\mu^{\top} x+\Phi^{-1}(p) \sqrt{x^{\top} \Sigma x} \leq d,
$$

where $\Phi^{-1}(p)$ is the $p$-quantile of the standard normal distribution. If $p \geq 0.5$, as it is usual in practice, then $\Phi^{-1}(p) \geq 0$, and, since $\sqrt{x^{\top} \Sigma x}$ is a convex function, the feasible set determined by (6) is a second-order cone constraint defining a convex feasible region. In a more general setting, the probability distribution of $\xi^{\top} x$ is often mixed or partially known and the value of the $p$-quantile of the distribution is unknown. The value of this latter can be approximated by using the well-known Cantelli, Chebychev [5] and Camp-Meidell [17] probability inequalities. Henrion [12] showed that the feasible set defined by the individual (i.e., one row) chance constraint $\mathbb{P}\left(\xi^{\top} h(x) \leq d\right) \geq p$ is convex if all the components of $h(x)$ are nonnegative and convex, $p \geq 0.5, \xi$ has an elliptical symmetric distribution, and the parameters of the distribution are nonnegative.

The complexity of stochastic problems with random technology matrix is further compounded as the number $r$ of inequalities that must hold jointly with a large probability level increases. Very few results are known for constraints with multi-row random technology matrix. Prékopa [26], Henrion and Strugarek [13], and Prékopa et al. [29] have studied the convexity properties of the feasible set $G=\{x: \mathbb{P}(T x \leq d) \geq p\}$ when each row $T_{i}$ of $T$ follows a continuous distribution. For instance, Prékopa [26] showed that $G$ is a convex set if all covariance and cross-covariance matrices of the columns and rows of $T$ are proportional to each other. Even if the rows $T_{i}$ are independent, the convexity of the feasible set defined by (3) can only be guaranteed under restrictive assumptions and is due to the fact that the product of quasi-concave functions is not necessarily quasi-concave [29]. Henrion and Strugarek [13] showed that the joint probabilistic constraint $\mathbb{P}\left(h_{i}(x) \geq \xi_{i}, i=1, \ldots, r\right) \geq p$ defines a convex feasible area if $h_{i}(x)$ is $\left(-d_{i}\right)$-concave and $\xi_{i}, i=1, \ldots, r$ are independent random variables with $\left(d_{i}+1\right)$ decreasing densities for some $d_{i}>0$ for large values of $p$. Considering a Gaussian technology matrix in (3), Van Ackooij et al. [33] designed an efficient method to compute the gradients and value of the multivariate Gaussian distribution functions with the code developed by Genz [9]. A possible approach is to approximate a multi-row chance constraint with random technology matrix (3) with individual chance 
constraints (7). This can be done by using, for example, Boole's inequality and requiring that:

$$
\begin{gathered}
\mathbb{P}\left(T_{i} x \leq d_{i}\right) \geq p_{i}, i=1, \ldots, r \\
\sum_{i=1}^{r} p_{i}-(r-1) \geq p \\
0 \leq p_{i} \leq p .
\end{gathered}
$$

However, this approach provides a very conservative solution.

In this paper, we consider the case in which the elements $t_{i j}$ of $T$ are discretely distributed. Discrete distributions are employed in multiple applications and are often created through sampling as approximations of the underlying distribution. Tanner and Ntaimo [32] propose a large-scale mixed integer programming reformulation for SRTM, derive the so-called irreducibly infeasible subsystem optimality cuts and solve the strengthened problem with a specialized branch-and-cut algorithm. Computational results are reported for instances of the optimal vaccine allocation problem in which the random technology matrix has one row. Ruszczyński [30] derives cutting planes based on precedence knapsack constraints which he uses within a branch-and-cut solution method. Beraldi and Bruni [3] consider the probabilistic set covering problem, in which random variables are present in the technology matrix and uncertainty is represented by a finite set of scenarios. The reformulated large-scale integer problem including knapsack constraints is solved with a specialized branch-and-bound algorithm.

The main objective of this paper is to develop a new modeling and exact solution method for a class of particularly complex stochastic programming problems. More precisely, we study stochastic problems that include a joint probabilistic constraint in which the elements of the multi-row random technology matrix are discretely distributed. Our modeling approach involves the binarization of the probability distribution and is carried out by using a set of threshold values, called cut points. The binarization process generates binary images for the realizations of the random variables and the set of recombinations, each of which is a vector defining possibly sufficient conditions for the probabilistic constraint to hold. The binarization is done in such a way that we can derive a threshold partially defined Boolean function (pdBf) representing the probabilistic constraint (3). The pdBf is defined with respect to the binary projections of the recombinations and consistently separates the $p$-sufficient recombinations from the $p$-insufficient ones. A key and novel contribution of this approach is to derive a tight threshold Boolean minorant for the pdBf representing the probabilistic constraint. Any separating structure of the tight threshold Boolean minorant defines sufficient conditions for the probability of $T x \leq d$ to be at least equal to $p$ and takes the form of a system of linear constraints.

The separating structure of the tight threshold Boolean minorant is a compact and computationally effective way to represent the pdBf. We use it to derive three new deterministic formulations equivalent to the probabilistically constrained problem SRTM. A crucial feature of these three new integer formulations is that the number of integer variables is equal to the number of cut points used in the binarization process and does not depend on the number of scenarios used to represent uncertainty. We also derive a set of strengthening valid inequalities for the reformulated problems. The computational experiments 
are based on multiple instances of the stochastic capital rationing problem and are used to analyze the computational efficiency of four algorithmic methods. The tests reveal that our mixed-integer reformulations make it possible to solve very fast and to optimality instances of the stochastic capital rationing problem in which the uncertainty is present in the rows of the technology matrix and is represented by a very large number of scenarios. The introduction of the proposed valid inequalities enables to further accelerate the solution process.

In Section 2, we describe the binarization process and the derivation of the pdBf representing (3). The method extends the Boolean framework proposed in $[18,19]$ to handle probabilistic constraints with random technology matrix. Section 3 describes the properties of the pdBf and introduces the concepts of extension and minorant of a Boolean function. It also characterizes the separating structure of the tight threshold minorant for the pdBf representing the probabilistic constraint (3). In Section 4, we derive three new reformulations equivalent to the stochastic programming problem SRTM. Section 5 describes the computational results, while Section 6 provides concluding remarks.

\section{Combinatorial Modeling Framework}

In this section, we present the binarization process / Boolean modeling framework used to reformulate probabilistic programming problems with multi-row and random technology matrix. The modeling framework involves the following steps: (i) the construction of the set of recombinations, and (ii) the binarization of the probability distribution. A variant of the proposed binarization process is used in $[18,19]$ for joint probabilistic constraints with random right-hand sides and deterministic random technology matrix. In [18], a $p$-efficient point [27] is represented as a conjunction of literals, called $e^{p}$ pattern, and mathematical programming problems for the generation of such patterns are proposed. In [19], Lejeune introduces the concept of a $p$-pattern that defines sufficient conditions for a chance constraint with random right-hand side to hold. An MIP reformulation equivalent to the associated stochastic problem is proposed and several heuristics providing high-quality $p$-patterns are evaluated.

\subsection{Construction of Set of Recombinations}

We denote by $\Omega$ the support set of $\xi$ : $\Omega$ contains the possible realizations of the $|J|$-random vector $\xi$ with distribution function $F$. A realization $k$ is represented by the $|J|$-deterministic numerical vector $\omega^{k}$. We distinguish $p$-sufficient and $p$-insufficient realizations.

Definition 1 [18] A realization $k$ is called $p$-sufficient if and only if $F\left(\omega^{k}\right) \geq p$ and is $p$-insufficient if $F\left(\omega^{k}\right)<p$.

As indicated by its name, a $p$-sufficient realization defines sufficient conditions for the probabilistic constraint (3) to hold. Indeed, if $k$ is $p$-sufficient, we have $F\left(\omega^{k}\right) \geq p$, and, thus,

$$
\omega^{k} x \leq d \quad \Rightarrow \quad \mathbb{P}(T x \leq d) \geq p
$$


We obtain a partition of the set $\Omega$ of realizations into two disjoint sets of $p$-sufficient $\Omega^{+}=\left\{k \in \Omega: F\left(\omega^{k}\right) \geq p\right\}$ and $p$-insufficient $\Omega^{-}=\Omega \backslash \Omega^{+}$realizations. To obtain the optimal solution for problem SRTM, we must not only take into account the realizations $k \in \Omega$ of the random vector, but we should also consider all points that can be $p$-sufficient and that we shall call recombinations. We denote by $F_{j}$ the marginal probability distribution of $\xi_{j}$ and by $F_{j}^{-1}(p)$ its $p$-quantile. The inequalities

$$
\omega_{j}^{k} \geq F_{j}^{-1}(p), j=1, \ldots,|J|
$$

are based on the univariate quantile rule and represent necessary, although not necessarily sufficient, conditions for the probabilistic constraint (3) to hold: $\mathbb{P}(T x \leq d) \geq p \Rightarrow \omega_{j}^{k} \geq F_{j}^{-1}(p), j=1, \ldots,|J|$. The sets $Z_{j}$ are given by

$$
Z_{j}=\left\{\omega_{j}^{k}: F_{j}\left(\omega_{j}^{k}\right) \geq p, k \in \Omega, j=1, \ldots,|J|,\right\}
$$

and their direct product $[18,28]$

$$
\bar{\Omega}=Z_{1} \times \ldots \times Z_{j} \times \ldots \times Z_{|J|},
$$

provides the set $\bar{\Omega}$ of recombinations that provides the exhaustive list of the points that can possibly be $p$ sufficient. Each $k \in \bar{\Omega}$ is called a recombination and is represented by a numerical vector $\omega^{k}$ satisfying the $|J|$ conditions defined by (11). A recombination $k \in \bar{\Omega}=\bar{\Omega}^{+} \cup \bar{\Omega}^{-}$can be $p$-sufficient or $p$-insufficient, and the disjoint sets $\bar{\Omega}^{+}=\left\{k \in \bar{\Omega}^{+}: F\left(\omega^{k}\right) \geq p\right\}$ and $\bar{\Omega}^{-}=\left\{k \in \bar{\Omega}^{-}: F\left(\omega^{k}\right)<p\right\}$ are respectively called sets of $p$-sufficient and $p$-insufficient recombinations. A recombination can be a realization of the random vector, but it is not necessarily the case. All $p$-sufficient realizations are included in the set of p-sufficient recombinations: $\Omega^{+} \subseteq \bar{\Omega}^{+}$.

\subsection{Binarization Process}

Using cut points [6], we shall now proceed to the binarization of the probability distribution and the set $\bar{\Omega}$. We denote by $n=\sum_{j \in J} n_{j}$ the sum of the number $n_{j}$ of cut points for each component $\xi_{j}$. The notation $\{0,1\}^{n}$ refers to the $n$-dimensional unit cube.

Definition 2 [18] The binarization process is the mapping $\mathbb{R}^{|J|} \rightarrow\{0,1\}^{n}$ of a numerical vector $\omega^{k}$ into a binary one $\beta^{k}=\left[\beta_{11}^{k}, \ldots, \beta_{1 n_{1}}^{k}, \ldots, \beta_{j l}^{k}, \ldots, \beta_{j n_{j}}^{k}, \ldots\right]$, such that the value of each component $\beta_{j l}^{k}$ is defined with respect to a cut point $c_{j l}$ as follows:

$$
\beta_{j l}^{k}=\left\{\begin{array}{ll}
1 & \text { if } \omega_{j}^{k} \geq c_{j l} \\
0 & \text { otherwise }
\end{array},\right.
$$

where $c_{j l}$ denotes the $l^{\text {th }}$ cut point associated with component $\xi_{j}$,

$$
l^{\prime}<l \Rightarrow c_{j l^{\prime}}<c_{j l}, j \in J, l=1, \ldots, n_{j} .
$$

Definition 3 A Boolean vector is called relevant if it is the binary mapping of a recombination. 
The binarization of $\bar{\Omega}$ provides the set $\bar{\Omega}_{B} \subseteq\{0,1\}^{n}$ called set of relevant Boolean vectors. Note that the binarization process arranges the cut points in ascending order (15).

Definition 4 A set of Boolean vectors is called regularized with respect to a group (i.e., ordered subset) $G_{j}=\left(\beta_{j 1}, \ldots, \beta_{j n_{j}}\right)$ of Boolean variables if every vector $\beta^{k}$ satisfies the following conditions:

$$
\begin{aligned}
& \beta_{j l}^{k}=1 \Rightarrow \beta_{j l^{\prime}}^{k}=1, l^{\prime}<l \\
& \beta_{j l}^{k}=0 \Rightarrow \beta_{j l^{\prime}}^{k}=0, l^{\prime}>l .
\end{aligned}
$$

Lemma 5 is a direct consequence of Definitions 2 and 4.

Lemma 5 The binarization process described in Definition 2 generates a regularized set of Boolean vectors, i.e., for every component $\xi_{j}, j \in J$, if $c_{j l^{\prime}}<c_{j l}$, then

$$
\beta_{j l}^{k} \leq \beta_{j l^{\prime}}^{k}, j \in J, k \in \Omega .
$$

Any vector $\beta^{k} \in\{0,1\}^{n}$ such that $\beta_{j l}^{k}>\beta_{j l^{\prime}}^{k}$ for any $l>l^{\prime}, j \in J$ does not correspond to any realization in the original numerical space and is therefore called not possible.

We shall define a set of cut points, such that the binarization process (15) preserves the disjointedness between the binary projections $\bar{\Omega}_{B}^{+}$and $\bar{\Omega}_{B}^{-}$of $\bar{\Omega}^{+}$and $\bar{\Omega}^{-}\left(\bar{\Omega}_{B}=\bar{\Omega}_{B}^{+} \cup \bar{\Omega}_{B}^{-}\right)$. We shall accomplish this by using the sufficient-equivalent [18] consistent set of cut points $C^{e}$.

Definition 6 The sufficient-equivalent consistent set of cut points is given by:

$$
C^{e}=\bigcup_{j=1}^{|J|} C_{j}, \quad \text { where } C_{j}=\left\{\omega_{j}^{k}: k \in \Omega^{+}\right\} .
$$

This ensures that no pair of $p$-sufficient and $p$-insufficient recombinations can have the same binary image. The set of relevant Boolean vectors is partitioned into the disjoint sets of $p$-sufficient $\bar{\Omega}_{B}^{+}$and $p$ insufficient $\bar{\Omega}_{B}^{-}$relevant Boolean vectors. Note that there is a one-to-one mapping between the numerical $\omega^{k}$ and the binary $\beta^{k}$ vectors associated with a recombination $k$ if the binarization process is carried out with the sufficient-equivalent set of cut points (19).

The binarization permits the derivation of a partially defined Boolean function (pdBf) representing the combination of the binary images $\beta^{k}$ of the recombinations $k$ with the prescribed probability level $p$.

Definition 7 Given two disjoint subsets $\bar{\Omega}_{B}^{+}, \bar{\Omega}_{B}^{-}: \bar{\Omega}_{B}^{+} \cup \bar{\Omega}_{B}^{-}=\bar{\Omega}_{B} \subseteq\{0,1\}^{n}, g\left(\bar{\Omega}_{B}^{+}, \bar{\Omega}_{B}^{-}\right)$is a partially defined Boolean function (pdBf) with the sets of true points $\bar{\Omega}_{B}^{+}$and false points $\bar{\Omega}_{B}^{-}$.

In this pdBf, the set of $p$-sufficient recombinations is the set of true points and the set of $p$-insufficient recombinations is the set of false points. Observe that the binarization process (Definition 2) and the intra-group regularity of the pdBf $g\left(\bar{\Omega}_{B}^{+}, \bar{\Omega}_{B}^{-}\right)$(Definition 4) imply that some vectors of the $n$-dimensional cube $\{0,1\}^{n}$ do not correspond to any point in the original numerical space and are not possible. For example, the binary vector $\left(\beta_{11}, \beta_{12}, \beta_{21}, \beta_{22}\right)=(0,1,1,0)$ is not possible, as (14)-(15) does not allow for $\beta_{11}$ to be strictly smaller than $\beta_{12}$. 
Theorem 8 Consider the binarization process obtained with $C^{e}$. The set $\bar{\Omega}_{B}$ of relevant Boolean vectors is the binary projection of $\bar{\Omega}$ and is given by

$$
\bar{\Omega}_{B}=\left\{k: \beta^{k} \in\{0,1\}^{n}: \beta_{j 1}^{k}=1, \beta_{j l}^{k} \leq \beta_{j l-1}^{k}, j \in J, l=2, \ldots, n_{j}\right\} .
$$

Proof. Consider an arbitrary point $k$ and the associated numerical $\omega^{k}$ and binary $\beta^{k}$ vectors. It follows from the univariate quantile rule (11) that $k$ is a recombination if and only if:

$$
\omega_{j}^{k} \geq \min \left\{\omega_{j}^{k}: k \in \Omega^{+}\right\}=\min \left\{\omega_{j}^{k}: \omega_{j}^{k} \in C_{j}\right\}=c_{j 1} \text { for any } j \in J \Leftrightarrow \beta_{j 1}^{k}=1 \text { for any } j \in J .
$$

The first equality follows from (19). The equivalence relationship is a consequence of the binarization process (14). The constraints $\beta_{j l}^{k} \leq \beta_{j l-1}^{k}$ in (20) follow from the regularization property (18).

We shall use the stochastic programming problem (22) with multi-row random technology matrix to illustrate our method:

$$
\begin{aligned}
& \max 2 x_{1}+x_{2}+x_{3} \\
& \text { subject to } \mathbb{P}\left\{\begin{array}{l}
2 \xi_{1} x_{1}+3 \xi_{2} x_{2}-25 \leq 0 \\
3 \xi_{1} x_{1}+6 \xi_{3} x_{3}-32 \leq 0
\end{array}\right\} \geq 0.7 \\
& x_{1}, x_{2}, x_{3} \geq 0
\end{aligned}
$$

where $s_{11}=2, s_{12}=s_{21}=3, s_{13}=s_{22}=0, s_{23}=6$, the random vectors are $T_{1}=\left[2 \xi_{1}, 3 \xi_{2}, 0\right]$ and $T_{2}=\left[3 \xi_{1}, 0,6 \xi_{3}\right]$. The cumulative probability distribution $F$ of the random variable $\xi=\left[\xi_{1}, \xi_{2}, \xi_{3}\right]$ is given in Table 1 . The realizations $\omega^{k}=\left[\omega_{1}^{k}, \omega_{2}^{k}, \omega_{3}^{k}\right]$ of $\xi$ are equally likely.

Table 1: Probability Distribution

\begin{tabular}{c||c|c|c|c|c|c|c|c|c|c}
$k$ & 1 & 2 & 3 & 4 & 5 & 6 & 7 & 8 & 9 & 10 \\
\hline$\omega_{1}^{k}$ & 7 & 1 & 1 & 3 & 2 & 3 & 5 & 7 & 7 & 7 \\
$\omega_{2}^{k}$ & 10 & 2 & 3 & 5 & 4 & 5 & 6 & 2 & 6 & 5 \\
$\omega_{3}^{k}$ & 2 & 1 & 3 & 2 & 1 & 1 & 3 & 4 & 3 & 5 \\
\hline$F\left(\omega^{k}\right)$ & 0.5 & 0.1 & 0.2 & 0.4 & 0.2 & 0.3 & 0.6 & 0.2 & 0.8 & 0.7
\end{tabular}

The sufficient-equivalent set of cut points is:

$$
C^{e}=\left\{c_{11}=7 ; c_{21}=5 ; c_{22}=6 ; c_{23}=10 ; c_{31}=3 ; c_{32}=4 ; c_{33}=5\right\} .
$$

Table 2 displays the recombinations and their binary images (i.e., relevant Boolean vectors) obtained with $C^{e}$.

It is easy to see that the numerical representation $\Omega^{+}$and $\Omega^{-}$of the sets of sufficient and insufficient numerical realizations are not linearly separable. Indeed, consider the two numerical insufficient realizations $\omega^{1}, \omega^{8} \in \Omega^{-}$. The convex combination $0.5 \omega^{1}+0.5 \omega^{8}$ is $\omega^{9} \in \Omega^{+}$, which shows that the convex hulls of $\Omega^{+}$and $\Omega^{-}$are not disjoint and indicates the non-convexity of problem (22).

\section{Extension and Minorant of pdBf: Functional Forms and Properties}

We shall now investigate how the $\operatorname{pdBf} g\left(\bar{\Omega}_{B}^{+}, \bar{\Omega}_{B}^{-}\right)$representing $(F, p)$ can be conveniently and efficiently used to solve problem SRTM. With that objective in mind, we introduce the concepts of an extension and 
Table 2: Reformulations and Relevant Boolean Vectors

\begin{tabular}{|c|c|c|c|c|c|c|c|c|c|c|c|}
\hline & \multicolumn{3}{|c|}{ Numerical Vectors } & \multicolumn{7}{|c|}{ Boolean Vectors } & Relevant Boolean Sets \\
\hline$k$ & $\omega_{1}^{k}$ & $\omega_{2}^{k}$ & $\omega_{3}^{k}$ & $\beta_{11}^{k}$ & $\beta_{21}^{k}$ & $\beta_{22}^{k}$ & $\beta_{23}^{k}$ & $\beta_{31}^{k}$ & $\beta_{32}^{k}$ & $\beta_{33}^{k}$ & \\
\hline 11 & 7 & 5 & 3 & 1 & 1 & 0 & 0 & 1 & 0 & 0 & \multirow{2}{*}{$\bar{\Omega}_{B}^{-}$} \\
\hline 12 & 7 & 5 & 4 & 1 & 1 & 0 & 0 & 1 & 1 & 0 & \\
\hline 9 & 7 & 6 & 3 & 1 & 1 & 1 & 0 & 1 & 0 & 0 & \multirow{7}{*}{$\bar{\Omega}_{B}^{+}$} \\
\hline 10 & 7 & 5 & 5 & 1 & 1 & 0 & 0 & 1 & 1 & 1 & \\
\hline 13 & 7 & 6 & 4 & 1 & 1 & 1 & 0 & 1 & 1 & 0 & \\
\hline 14 & 7 & 6 & 5 & 1 & 1 & 1 & 0 & 1 & 1 & 1 & \\
\hline 15 & 7 & 10 & 3 & 1 & 1 & 1 & 1 & 1 & 0 & 0 & \\
\hline 16 & 7 & 10 & 4 & 1 & 1 & 1 & 1 & 1 & 1 & 0 & \\
\hline 17 & 7 & 10 & 5 & 1 & 1 & 1 & 1 & 1 & 1 & 1 & \\
\hline & & & & \multicolumn{7}{|c|}{ Cut Points } & \\
\hline & & & & 7 & 5 & 6 & 10 & 3 & 4 & 5 & \\
\hline
\end{tabular}

minorant of a pdBf and we continue with the characterization of the Boolean functional form under which $g\left(\bar{\Omega}_{B}^{+}, \bar{\Omega}_{B}^{-}\right)$can be represented. This is instrumental for the derivation of a deterministic reformulation of problem SRTM.

\subsection{Boolean functional Form and Extension}

Definition 9 [8] A Boolean function $f$ of $n$ variables is a mapping $f:\{0,1\}^{n} \rightarrow\{0,1\}$. A point $X=\left(x_{1}, x_{2}, \ldots, x_{n}\right) \in\{0,1\}^{n}$ is a true point (resp., false point) of $f$ if $f(X)=1$ (resp., $\left.f(X)=0\right)$. We denote by $\mathcal{T}(f)$ and $\mathcal{F}(f)$ the disjoint sets of respectively true and false points of $f$.

Note that a Boolean function is a special case of a pdBf (Definition 7): any pdBf $g\left(\bar{\Omega}_{B}^{+}, \bar{\Omega}_{B}^{-}\right)$such that $\bar{\Omega}_{B}^{+} \cup \bar{\Omega}_{B}^{-}=\{0,1\}^{n}$ is a Boolean function.

Definition 10 A Boolean function $f$ is called an extension of a pdBf $g\left(\bar{\Omega}_{B}^{+}, \bar{\Omega}_{B}^{-}\right)$if $\bar{\Omega}_{B}^{+} \subseteq \mathcal{T}(f)$ and $\bar{\Omega}_{B}^{-} \subseteq \mathcal{F}(f)$.

We shall now consider several Boolean functional forms that extensions of pdBf can take.

Definition 11 [8] A Boolean function $f$ is positive (resp., negative) monotone in the binary variable $x_{l}, l \in\{1, \ldots, n\}$ if $f_{\mid x_{l}=0} \leq f_{\mid x_{l}=1}$ (resp., $f_{\mid x_{l}=1} \leq f_{\mid x_{l}=0}$ ) regardless of the values taken by the $(n-1)$ other $x_{l}^{\prime} \in\{0,1\}, l^{\prime} \in\{1, \ldots, n\} \backslash\{l\}$. A Boolean function $f$ is positive monotone (also called, increasing monotone or isotone) if it is positive in each of its variables.

The following result was derived in [18]:

Theorem 12 Any consistent pdBf representing $(F, p)$ can be extended as an isotone Boolean function. 
Definition 13 [8] A function $f:\{0,1\}^{n} \rightarrow\{0,1\}$ is a threshold Boolean function if, for all $\left(x_{1}, \ldots, x_{n}\right) \in$ $\{0,1\}^{n}$, there exists a vector of weights $w \in \mathbb{R}^{n}$ and a threshold $\theta \in \mathbb{R}$, such that

$$
f\left(x_{1}, \ldots, x_{n}\right)=1 \quad \text { if and only if } \sum_{l=1}^{n} w_{l} x_{l} \geq \theta .
$$

The hyperplane $\left\{x \in\{0,1\}^{n}: \sum_{l=1}^{n} w_{l} x_{l}=\theta\right\}$ is a separator for $f$ and the $(n+1)$-tuple $(w, \theta)$ is the separating structure for the threshold Boolean function $f$.

The problem of proving that a Boolean function is a threshold one is known as the threshold recognition or synthesis problem [22]. The threshold nature of a Boolean function can be identified numerically, in polynomial time, by solving a linear programming problem [8].

Theorem 14 The pdBf $g\left(\bar{\Omega}_{B}^{+}, \bar{\Omega}_{B}^{-}\right)$accepts a threshold Boolean extension if and only if the system of inequalities

$$
\begin{aligned}
& \sum_{j \in J} \sum_{l=1}^{n_{j}} w_{j l} \beta_{j l}^{k} \quad \geq \theta, \quad k \in \bar{\Omega}_{B}^{+} \\
& \sum_{j \in J} \sum_{l=1}^{n_{j}} w_{j l} \beta_{j l}^{k} \quad \leq \theta-1, \quad k \in \bar{\Omega}_{B}^{-}
\end{aligned}
$$

is feasible. If this is the case, the solution $\left(w_{11}, \ldots, w_{|J| n_{|J|}}, \theta\right)$ defines the separating structure of $g$.

The proof follows from Definition 13 by standard argument. Theorem 14 illustrates the simplicity and convenience of the representation of a threshold function which is fully characterized by the $(n+1)$-tuple $(w, \theta)$ defining its separating structure. This means that each $p$-sufficient (resp., $p$-insufficient) realization is also labeled as $p$-sufficient (resp., $p$-insufficient) by the linear rule defined by the separating structure $(w, \theta)$. Sufficient conditions for satisfying the constraint (3) can thus be derived from the knowledge of the separating structure.

Consider problem (22) and the set $\bar{\Omega}_{B}$ of relevant Boolean vectors displayed in Table 2 . Note that, using (21), we dropped each realization $k$ with Boolean vector $\beta^{k}$ such that $\beta_{j 1}^{k}=0$ for at least one $j$, since (21) implies such a realization $k$ cannot be $p$-sufficient. Minimizing the value of the threshold $\theta$, 
the threshold recognition problem for our example reads:

$$
\begin{aligned}
& \min _{w, \theta} \theta \\
& \text { s. to } w_{11}+w_{21}+w_{22}+w_{31} \geq \theta \\
& (k=9) \\
& w_{11}+w_{21}+w_{31}+w_{32}+w_{33} \geq \theta \quad(k=10) \\
& w_{11}+w_{21}+w_{22}+w_{31}+w_{32} \geq \theta \quad(k=13) \\
& w_{11}+w_{21}+w_{22}+w_{31}+w_{32}+w_{33} \geq \theta \quad(k=14) \\
& w_{11}+w_{21}+w_{22}+w_{23}+w_{31} \geq \theta \quad(k=15) \\
& w_{11}+w_{21}+w_{22}+w_{23}+w_{31}+w_{32} \geq \theta \quad(k=16) \\
& w_{11}+w_{21}+w_{22}+w_{23}+w_{31}+w_{32}+w_{33} \geq \theta \quad(k=17) \\
& w_{11}+w_{21}+w_{31} \leq \theta-1 \quad(k=11) \\
& w_{11}+w_{21}+w_{31}+w_{32} \leq \theta-1 \quad(k=12)
\end{aligned}
$$

The optimal solution is $\left(w^{*}, \theta^{*}\right)=(0,0,1,0,0,0,1,1)$. Note that $\beta_{j 1}^{k}=1, k \in \bar{\Omega}_{B}$. Hence, the Boolean variables $\beta_{j 1}, j=1,2,3$ do not play any role in the derivation of the separating structure and we do not need to associate a weight to them. Observe that the system of linear inequalities (24)-(25) is not always feasible.

Theorem 15 The pdBf $g\left(\bar{\Omega}_{B}^{+}, \bar{\Omega}_{B}^{-}\right)$may not be extendable as a threshold Boolean function.

Proof. Let $\xi$ be a four-dimensional vector with two cut points associated with each component. Consider the

1. sufficient Boolean vectors $\beta^{1}=(1,1,1,1,1,0,1,0)$ and $\beta^{2}=(1,0,1,0,1,1,1,1), k=1,2 \in \Omega^{+}$, and

2. insufficient Boolean vectors $\beta^{3}=(1,1,1,0,1,0,1,1)$ and $\beta^{4}=(1,0,1,1,1,1,1,0), k=3,4 \in \Omega^{-}$.

Observe that the above set of Boolean vectors is valid for the problem at hand, since $\beta_{j 1}^{k}=1, j=1, \ldots, 4$ and that there is no $k \in \Omega^{-}$such that $\beta^{k} \geq \beta^{k^{\prime}}$ for any $k^{\prime} \in \Omega^{+}$.

Assume that $g\left(\bar{\Omega}_{B}^{+}, \bar{\Omega}_{B}^{-}\right)$can be extended as a threshold Boolean function. In that case, theres exists a tuple $(w, \theta)$ satisfying the system of linear inequalities:

$$
\begin{cases}w_{11}+w_{12}+w_{21}+w_{22}+w_{31}+w_{41} \geq \theta & (k=1) \\ w_{11}+w_{21}+w_{31}+w_{32}+w_{41}+w_{42} \geq \theta & (k=2) \\ w_{11}+w_{12}+w_{21}+w_{31}+w_{41}+w_{42} \leq \theta-1 & (k=3) \\ w_{11}+w_{21}+w_{22}+w_{31}+w_{32}+w_{41} \leq \theta-1 & (k=4)\end{cases}
$$

The feasibility of the above system requires that the system

$$
\begin{cases}2 w_{11}+w_{12}+2 w_{21}+w_{22}+2 w_{31}++w_{31}+2 w_{41}+w_{42} \geq 2 \theta & (k=1)+(k=2) \\ 2 w_{11}+w_{12}+2 w_{21}+w_{22}+2 w_{31}++w_{32}+2 w_{41}+w_{42} \leq 2 \theta-2 & (k=3)+(k=4)\end{cases}
$$


obtained by summing up the first two inequalities and the last two be feasible. This obviously leads to a contradiction and provides the desired result.

However, we do not need to derive an extension of $g\left(\bar{\Omega}_{B}^{+}, \bar{\Omega}_{B}^{-}\right)$. The knowledge of an extension $f$ for $g\left(\bar{\Omega}_{B}^{+}, \bar{\Omega}_{B}^{-}\right)$permits to derive linear inequalities representing sufficient conditions for the probabilistic constraint (3) to hold. To qualify as an extension for $g\left(\bar{\Omega}_{B}^{+}, \bar{\Omega}_{B}^{-}\right)$, it can be seen above that $f$ must meet strict conditions. An extension $f$ could very likely be such that we might not be able to extract the minimal conditions that must be met for (3) to be satisfied, thus impeding us to derive a deterministic formulation equivalent to SRTM. Thus, in order to identify tighter, possibly minimal conditions for constraint (3) to hold, we shall enlarge the class of Boolean functions we consider beyond that of extensions and characterize several forms of minorant for Boolean functions.

\subsection{Concept of Minorant}

We shall now introduce the concepts of minorant and tight minorant of a Boolean function.

Definition 16 [8] A Boolean function $f$ is a minorant of a pdBf $g\left(\bar{\Omega}_{B}^{+}, \bar{\Omega}_{B}^{-}\right)$if $\bar{\Omega}_{B}^{-} \subseteq \mathcal{F}(f)$.

A minorant is a very broad concept, as many functions whose structure is loosely tied to the $\mathrm{pdBf}$ $g\left(\bar{\Omega}_{B}^{+}, \bar{\Omega}_{B}^{-}\right)$could be a minorant of $g\left(\bar{\Omega}_{B}^{+}, \bar{\Omega}_{B}^{-}\right)$. For example, the constant 0 is a minorant for any pdBf. We shall now introduce the concept of tight minorant.

Definition 17 A Boolean function $f$ is a tight minorant of a pdBf $g\left(\bar{\Omega}_{B}^{+}, \bar{\Omega}_{B}^{-}\right)$if

1. $f$ is a minorant of $g\left(\bar{\Omega}_{B}^{+}, \bar{\Omega}_{B}^{-}\right)$, and

2. $\mathcal{T}(f) \cap \bar{\Omega}_{B}^{+} \neq \emptyset$.

Since a threshold Boolean function is characterized by a separating structure, Definition 17 leads to Lemma 18.

Lemma 18 A threshold Boolean function $f$ defined by the separating structure $(w, \theta)$ is a tight minorant of a pdBf $g\left(\bar{\Omega}_{B}^{+}, \bar{\Omega}_{B}^{-}\right)$if the system of inequalities

$$
\begin{array}{lr}
\sum_{j \in J} \sum_{l=1}^{n_{j}} w_{j l} \beta_{j l}^{k} \geq \theta, & \text { for at least one } k \in \bar{\Omega}_{B}^{+} \\
\sum_{j \in J} \sum_{l=1}^{n_{j}} w_{j l} \beta_{j l}^{k} \leq \theta-1, & k \in \bar{\Omega}_{B}^{-}
\end{array}
$$

has a feasible solution.

Observe that (26) is a disjunctive condition over the set $\bar{\Omega}_{B}^{+}$. 
Theorem 19 Given a pdBf $g\left(\bar{\Omega}_{B}^{+}, \bar{\Omega}_{B}^{-}\right)$that admits a positive monotone extension, there exists a tight minorant of $g\left(\bar{\Omega}_{B}^{+}, \bar{\Omega}_{B}^{-}\right)$that is threshold.

Proof. Since $g\left(\bar{\Omega}_{B}^{+}, \bar{\Omega}_{B}^{-}\right)$admits a positive monotone extension (Theorem 12), we know that:

- For any $k \in \bar{\Omega}_{B}^{+}$,

$$
\text { there is no } k^{\prime} \in \bar{\Omega}_{B}^{-} \text {such that } \beta^{k^{\prime}} \geq \beta^{k} \text {. }
$$

- For any $k \in \bar{\Omega}_{B}^{-}$,

$$
\text { there is no } k^{\prime} \in \bar{\Omega}_{B}^{+} \text {such that } \beta^{k^{\prime}} \leq \beta^{k} \text {. }
$$

Take an arbitrary $k^{*} \in \bar{\Omega}_{B}^{+}$and construct a threshold function $f$ (Definition 13) whose separating structure has weights $w$ taking value 0 or 1 such that

$$
w_{j l}=\beta_{j l}^{k^{*}} \quad \text { and } \quad \theta=\sum_{j \in J} \sum_{l=1}^{n_{j}} \beta_{j l}^{k^{*}} .
$$

By construction,

$$
\sum_{j \in J} \sum_{l=1}^{n_{j}} w_{j l} \beta_{j l}^{k^{*}}=\theta,
$$

and $f\left(\beta^{k^{*}}\right)=1$. All what is left to show is that $f\left(\beta^{k}\right)=0$ for any $k \in \bar{\Omega}_{B}^{-}$.

Take an arbitrary point $k^{\prime} \in \bar{\Omega}_{B}^{-}$and proceed to a componentwise comparison with $\beta^{k^{*}}$. Since $\bar{\Omega}_{B}^{+}$and $\bar{\Omega}_{B}^{-}$ are disjoint, it follows that $\beta^{k^{*}}$ and $\beta^{k^{\prime}}$ differ on at least one coordinate:

$$
\sum_{j \in J} \sum_{l=1}^{n_{j}}\left|\beta_{j l}^{k^{*}}-\beta_{j l}^{k^{\prime}}\right| \geq 1 .
$$

Since $g$ is positive monotone (29) and $\beta^{k^{*}} \neq \beta^{k^{\prime}}$ since $\bar{\Omega}_{B}^{+}$and $\bar{\Omega}_{B}^{-}$are disjoint, we have that $\beta_{j l}^{k^{\prime}}<\beta_{j l}^{k^{*}}$ on at least one coordinate $j l$. This implies that $\beta^{k^{\prime}}$ takes value 0 for at least one $j l$ on which $\beta^{k^{*}}$ is equal to one. Therefore, by construction of $\beta^{k^{*}}$ (30) and of the separating structure (31) of $f$, it follows that

$$
\sum_{j \in J} \sum_{l=1}^{n_{j}} w_{j l} \beta_{j l}^{k^{\prime}}=\sum_{j l::_{j l}^{k^{*}}=1} \beta_{j l}^{k^{\prime}} \leq \theta-1,
$$

which was set out to prove.

The inequalities (27) mean that each $p$-insufficient realization is also defined as $p$-insufficient by the separating structure $(w, \theta)$ characterizing the tight minorant $f$. Lemma 20 follows.

Lemma 20 The threshold tight minorant derived in the proof of Theorem 19 has an integral separating structure $\left(w_{11}, \ldots, w_{|J| n_{|J|}}, \theta\right) \in \mathbb{Z}_{+}^{n} \times \mathbb{Z}_{+}$and is isotone by construction, i.e., $w_{j l} \geq 0, j \in J, l=1, \ldots, n_{j}$. 
A Boolean function $f$ is said to depend on a Boolean variable $\beta_{j l}$ if there are two Boolean vectors that differ only on $\beta_{j l}$ and for which the values of the Boolean function are not the same. Note that a threshold function does not depend on $\beta_{j l}$ if the corresponding weight $w_{j l}$ is zero in the separating structure of $f$.

We shall now introduce the concept of irredundant tight minorant.

Definition 21 A threshold tight minorant is called irredundant if it has the minimum number of weights $w_{j l}$ with non-zero value.

Theorem 22 defines the number of non-zero weights in an irredundant threshold tight minorant $f$ of $g\left(\bar{\Omega}_{B}^{+}, \bar{\Omega}_{B}^{-}\right)$, and a set of conditions allowing for their generation.

Theorem 22 An irredundant threshold tight minorant $f$ of $g\left(\bar{\Omega}_{B}^{+}, \bar{\Omega}_{B}^{-}\right)$contains $|J|$ non-zero weights. Every feasible solution of the system of inequalities

$$
\begin{array}{lr}
\sum_{j \in J} \sum_{l=1}^{n_{j}} w_{j l} \beta_{j l}^{k} \leq|J|-1, & k \in \bar{\Omega}_{B}^{-} \\
\sum_{l=1}^{n_{j}} w_{j l}=1, & j \in J \\
w_{j l} \in\{0,1\}, & j \in J, l=1, \ldots, n_{j}
\end{array}
$$

defines an irredundant threshold tight minorant $f$ with integral separating structure $(w,|J|) \in\{0,1\}^{n} \times \mathbb{Z}_{+}$. Every point $k$ such that $\sum_{j \in J} \sum_{l=1}^{n_{j}} w_{j l} \beta_{j l}^{k}=|J|$ belongs to $\bar{\Omega}_{B}^{+}$.

Proof. For a joint chance constraint (3) to hold, at least one condition must be imposed on each component of $\xi$ (see, e.g., [27, 30]). Thus, the minimal number of non-zero weights in a threshold tight minorant of $g\left(\bar{\Omega}_{B}^{+}, \bar{\Omega}_{B}^{-}\right)$is $|J|$. This explains why, in the right-hand side of (32), we substitute $(|J|-1)$ in (32) for $\theta$ as in (27).

Consider the extreme recombination $k^{\prime}$ with $\beta^{k^{\prime}}$ being constant 1 , i.e., $\beta_{j l}^{k^{\prime}}=1, l=1, \ldots, n_{j}, j \in J$. Clearly, $F\left(\omega^{k^{\prime}}\right)=1$ and $k^{\prime} \in \bar{\Omega}_{B}^{+}$. For any feasible solution for (32)-(34), we have

$$
\sum_{j \in J} \sum_{l=1}^{n_{j}} w_{j l} \beta_{j l}^{k^{\prime}}=\sum_{j \in J} \sum_{l=1}^{n_{j}} w_{j l} \geq \sum_{j \in J} 1=|J| .
$$

Thus, (26) is induced by (32)-(34) and is redundant.

Consider an arbitrary separating structure $(w,|J|)$ feasible for (32)-(34). All possible Boolean vectors belong either to the set $\bar{\Omega}_{B}^{+}$of relevant $p$-sufficient Boolean vectors, or the set $\bar{\Omega}_{B}^{-}$of relevant $p$-insufficient Boolean vectors, or the set of $\left(\Omega_{B}^{-} \backslash \bar{\Omega}_{B}^{-}\right)$of non-relevant $p$-insufficient realizations. Constraint (32) prevents any $k \in \bar{\Omega}_{B}^{-}$from satisfying (35):

$$
\sum_{j \in J} \sum_{l=1}^{n_{j}} w_{j l} \beta_{j l}^{k} \geq|J| .
$$


Any $k \in\left(\Omega_{B}^{-} \backslash \bar{\Omega}_{B}^{-}\right)$is such that $\beta_{j 1}^{k}=0$ for at least one $j \in J$. Combining this result with the regularization property (see (18) in Lemma 5), we have:

$$
\beta_{j l}^{k}=0, l=1, \ldots, n_{j} \text { for at least one } j k \in\left(\Omega_{B}^{-} \backslash \bar{\Omega}_{B}^{-}\right)
$$

Therefore,

$$
\sum_{l=1}^{n_{j}} w_{j l} \beta_{j l}^{k}=0, \text { for at least one } j \in J, k \in\left(\Omega_{B}^{-} \backslash \bar{\Omega}_{B}^{-}\right)
$$

which implies that

$$
\sum_{j \in J} \sum_{l=1}^{n_{j}} w_{j l} \beta_{j l}^{k} \leq|J|-1, k \in\left(\Omega_{B}^{-} \backslash \bar{\Omega}_{B}^{-}\right),
$$

and thus that (35) does not hold for any $k \in\left(\Omega_{B}^{-} \backslash \bar{\Omega}_{B}^{-}\right)$. Hence, any $k$ for which (35) holds belongs to $\bar{\Omega}_{B}^{+}$.

Theorem 22 shows that we do not need to include the disjunction (26) to generate a tight minorant of $g\left(\bar{\Omega}_{B}^{+}, \bar{\Omega}_{B}^{-}\right)$.

Theorem 23 Any feasible solution $w$ of the system of linear inequalities

$$
\begin{array}{lr}
\sum_{j \in J} \sum_{l=1}^{n_{j}} w_{j l} \beta_{j l}^{k} \leq|J|-1, & k \in \bar{\Omega}_{B}^{-} \\
\sum_{l=1}^{n_{j}} w_{j l}=1, & j \in J \\
0 \leq w_{j l} \leq 1, & j \in J, l=1, \ldots, n_{j}
\end{array}
$$

can be used to obtain an integral separating structure $(\bar{w},|J|) \in\{0,1\}^{n} \times \mathbb{Z}_{+}$for an irredundant threshold tight minorant $f$ of $g$ in the following way:

$$
\bar{w}_{j l_{j}^{*}}=1 \quad \text { and } \quad \bar{w}_{j l}=0, l_{j} \neq l_{j}^{*}, \quad \text { with } \quad l_{j}^{*}=\underset{l}{\operatorname{argmax}} w_{j l}>0, j \in J, l_{j}=1, \ldots, n_{j} .
$$

Proof. We have to show that constraints (32)-(34) hold for the separating structure $(\bar{w},|J|)$. It is straightforward to see that the rounding procedure (39) is such that (33)-(34) holds for $\bar{w}$. What is left to prove is that (32) holds for $\bar{w}$, which is equivalent to showing

$$
\sum_{j \in J} \sum_{l=1}^{n_{j}} \bar{w}_{j l} \beta_{j l}^{k} \leq \sum_{j \in J} \sum_{l=1}^{n_{j}} w_{j l} \beta_{j l}^{k}, \quad \forall k \in \bar{\Omega}_{B}^{-} \quad \Leftrightarrow \quad \sum_{j \in J} \sum_{l=1}^{l_{j}^{*}} \bar{w}_{j l} \beta_{j l}^{k} \leq \sum_{j \in J} \sum_{l=1}^{l_{j}^{*}} w_{j l} \beta_{j l}^{k}, \quad \forall k \in \bar{\Omega}_{B}^{-}
$$

for $\bar{w}$ derived with (39) from $w$ feasible for (36)-(38).

Consider one component $j \in J$ and let us show that:

$$
\sum_{l=1}^{l_{j}^{*}} \bar{w}_{j l} \beta_{j l}^{k} \leq \sum_{l=1}^{l_{j}^{*}} w_{j l} \beta_{j l}^{k}, \quad \forall k \in \bar{\Omega}_{B}^{-}
$$


If $\beta_{j l_{j}^{*}}^{k}=1$, then (39) implies that $\sum_{l=1}^{i_{j}^{*}} \bar{w}_{j l} \beta_{j l}^{k}=1$, and we have, from the regularization property (16), that:

$$
\beta_{j l}^{k}=1, \forall l<l_{j}^{*} \quad \text { and thus that } \quad \sum_{l=1}^{l_{j}^{*}} w_{j l} \beta_{j l}^{k}=\sum_{l=1}^{l_{j}^{*}} w_{j l}=1 .
$$

The last inequality follows from (39) and the fact that $w$ satisfies (37).

If $\beta_{j l_{j}^{*}}^{k}=0$, then

$$
\sum_{l=1}^{l_{j}^{*}} \bar{w}_{j l} \beta_{j l}^{k}=0 \leq \sum_{l=1}^{l_{j}^{*}} w_{j l} \beta_{j l}^{k} .
$$

Evidently, (42) and (43) imply (41). The same result is valid for any $j \in J$, which guarantees that (40) holds.

In our example, the system of inequalities (32)-(34) reads:

$$
\begin{aligned}
& w_{11}+w_{21}+w_{31} \leq 2 \\
& w_{11}+w_{21}+w_{31}+w_{32} \leq 2 \\
& w_{11}=1 \\
& w_{21}+w_{22}+w_{23}=1 \\
& w_{31}+w_{32}+w_{33}=1 \\
& w_{j l} \in\{0,1\}, \quad \forall j, l .
\end{aligned}
$$

Any feasible solution for (44)-(48), such as $\mathbf{w}=(1,0,1,0,1,0,0)$, defines the separating structure of an irredundant threshold tight minorant of $g\left(\bar{\Omega}_{B}^{+}, \bar{\Omega}_{B}^{-}\right)$. The above results are used in the Section 4 to derive deterministic approximations and equivalents for the original stochastic problem SRTM.

Theorem 24 [8] If the pdBf $g\left(\bar{\Omega}_{B}^{+}, \bar{\Omega}_{B}^{-}\right)$is a threshold function defined on $\{0,1\}^{n}$, then the set of separating structures is a full-dimensional convex cone in $\mathbb{R}^{n+1}$.

Proof. Let $\lambda, \mu \geq 0$ and $D=(w, \theta)$ and $D^{\prime}=\left(w^{\prime}, \theta^{\prime}\right)$ be two arbitrary separating structures of $g\left(\bar{\Omega}_{B}^{+}, \bar{\Omega}_{B}^{-}\right)$. It is straightforward that $\alpha D$ and $\alpha D+\mu D^{\prime}$ are also separating structures of $g$, which proves the above statement.

\section{Threshold Minorant Reformulation of Probabilistically Constrained Prob- lems}

In this section, we shall use the threshold structure of the tight minorant the $\operatorname{pdBf} g\left(\bar{\Omega}_{B}^{+}, \bar{\Omega}_{B}^{-}\right)$to reformulate the probabilistically constrained problem SRTM. New inner approximation and deterministic equivalent reformulations for SRTM are derived. 


\subsection{Inner Approximation Formulation}

In this section, we derive a linear programming formulation that constitutes an inner approximation of the stochastic problem SRTM. We recall that each component $t_{i j}=s_{i j} \xi_{j}$ of the matrix $T$ in (3) involves a stochastic component $\xi_{j}$.

Theorem 25 Let $(w,|J|)$ be a feasible solution for (36)-(38) and define $l_{j}^{*}$ with (39) for each $j \in J$. The linear deterministic constraints

$$
\sum_{j \in J} s_{i j} c_{j l_{j}^{*}} x_{j} \leq d_{i}, i=1, \ldots, r
$$

define sufficient conditions for $\mathbb{P}(T x \leq d)$ to be at least equal to $p$.

Proof. Let us take an arbitrary point feasible for (36)-(38) and construct the irredundant tight minorant $f^{*}$ with separating structure $(w,|J|)$ defined according to (39). It follows from Theorem 23 that the separating structure of $f^{*}$ is such that

$$
\sum_{j \in J} \beta_{j l_{j}^{*}}^{k} \leq|J|-1, k \in \bar{\Omega}_{B}^{-} \quad \text { and } \quad \sum_{j \in J} \beta_{j l_{j}^{*}}^{k} \geq|J| \quad \text { for at least one } k \in \bar{\Omega}_{B}^{+} .
$$

This underlines that the conditions imposed by $f^{*}$ (i.e., induced by its separating structure) are violated by each $k \in \bar{\Omega}_{B}^{-}$but hold for at least one $k \in \bar{\Omega}_{B}^{+}$. Using the definition of the binarization process (14)-(15), one can see that (51) is equivalent to:

$$
\left\{\begin{array}{l}
\omega_{j}^{k}<c_{j l^{*}} \beta_{j l_{j}^{*}}^{k}=c_{j l^{*}}, \quad \text { for at least one } j, \forall k \in \bar{\Omega}^{-} \\
\omega_{j}^{k} \geq c_{j l^{*}} \beta_{j l^{*}}^{k}=c_{j l^{*}}, \quad \forall j \in J, \text { for at least one } k \in \bar{\Omega}^{+}
\end{array} .\right.
$$

Further, the construction of the sufficient-equivalent set of cut points (see (19) in Definition 6) implies

$$
\omega_{j}^{k}=c_{j l^{*}}, \quad j \in J, \text { for one } k \in \bar{\Omega}^{+} .
$$

Let $k^{\prime} \in \bar{\Omega}^{+}$be the recombination for which (53) holds:

$$
\omega_{j}^{k^{\prime}}=c_{j l^{*}}, \quad j \in J
$$

Every component $t_{i j}$ of $T$ is defined by: $t_{i j}=s_{i j} \xi_{j}, i=1, \ldots, r, j \in J$. Thus, we have

$$
\mathbb{P}(T x \leq d)=\mathbb{P}\left(\sum_{j \in J} s_{i j} \xi_{j} x_{j} \leq d_{i}, i=1, \ldots, r\right) .
$$

Since $k^{\prime} \in \bar{\Omega}^{+}$, we have $\mathbb{P}\left(\xi \leq \omega^{k^{\prime}}\right) \geq p$ and

$$
\mathbb{P}\left(\sum_{j \in J} s_{i j} \xi_{j} x_{j} \leq \sum_{j \in J} s_{i j} \omega_{j}^{k^{\prime}} x_{j}, i=1, \ldots, r\right) \geq p
$$


given that $\xi_{j}, \omega_{j}^{k^{\prime}}, x_{j}$, and $s_{i j}$ are all non-negative. This, combined with (55), implies that:

$$
\sum_{j \in J} s_{i j} \omega_{j}^{k^{\prime}} x_{j} \leq d_{i}, i=1, \ldots, r \quad \Rightarrow \quad \mathbb{P}\left(\sum_{j \in J} s_{i j} \xi_{j} x_{j} \leq d_{i}, i=1, \ldots, r\right) \geq p .
$$

Using (54), we can rewrite (57) as

$$
\sum_{j \in J} s_{i j} c_{j l^{*}} x_{j} \leq d_{i}, i=1, \ldots, r \quad \Rightarrow \quad \mathbb{P}(T x \leq d) \geq p
$$

which was set out to prove.

Corollary 26 follows immediately from Theorem 25 .

Corollary 26 The linear program $\mathbf{L P}$

$$
\begin{aligned}
& \text { LP : } \quad \max q^{\top} x \\
& \text { subject to }(2) ;(4) ;(50)
\end{aligned}
$$

is an inner approximation of the probabilistically constrained problem SRTM. Its feasible set is included in the feasible set of SRTM.

Consider $\mathbf{w}=(1,0,1,0,1,0,0)$ which is feasible for (44)-(48) and defines the separating structure of an irredundant threshold tight minorant of $g\left(\bar{\Omega}_{B}^{+}, \bar{\Omega}_{B}^{-}\right)$. The corresponding constraints (50) are:

$$
\begin{aligned}
& 2 \cdot 7 \cdot x_{1}+3 \cdot 6 \cdot x_{2} \leq 25 \\
& 3 \cdot 7 \cdot x_{1}+6 \cdot 3 \cdot x_{3} \leq 32 .
\end{aligned}
$$

The associated linear programming problem $\mathbf{L P}$ is

$$
\begin{aligned}
\max & 2 x_{1}+x_{2}+x_{3} \\
\text { subject to } & (59)-(60) \\
& x_{1}, x_{2}, x_{3} \geq 0
\end{aligned}
$$

\subsection{Equivalent Formulations}

In this section, we build on the results of Section 4 to derive three new deterministic formulations that are equivalent to the stochastic problem SRTM. Instead of successively solving the system of linear inequalities (36)-(38) to derive a threshold tight minorant and to solve the resulting inner approximation linear programming problem $\mathbf{L P}$, we propose in this section to execute the two tasks concurrently through the solution of a deterministic problem equivalent to SRTM. 


\subsubsection{Bilinear Integer Programming Formulation}

We first reformulate SRTM as a bilinear integer programming problem.

Theorem 27 The quadratic integer optimization problem QDE

$$
\begin{array}{rll}
\text { QDE : max } & q^{\top} x & \\
\text { subject to } & (2) ;(4) ;(32) ;(33) ;(34) & \\
& \sum_{l=1}^{n_{j}} w_{j l} c_{j l}=y_{j}, & j \in J \\
& \sum_{j \in J} s_{i j} y_{j} x_{j} \leq d_{i}, & i=1, \ldots, r \\
& c_{j 1} \leq y_{j} \leq c_{j n_{j}}, & j \in J
\end{array}
$$

is equivalent to the probabilistically constrained problem SRTM.

Proof. (i) Any solution feasible for QDE is feasible for SRTM. Since constraints (32), (33) and (34) are part of the constraint set, we can use Theorem 22 that indicates that any $\hat{w} \in\{w:(32),(33),(34)\}$ defines the separating structure $(\hat{w},|J|)$ of an irredundant threshold tight minorant $f$. In other words, $G=\left\{k \in \bar{\Omega}_{B}: \sum_{j \in J} \sum_{l=1}^{n_{j}} \hat{w}_{j l} \beta_{j l}^{k}=|J|\right\} \subseteq \bar{\Omega}_{B}^{+}$.

Let $L=\left\{j l: \hat{w}_{j l}=1, j \in J, l=1, \ldots, n_{j}\right\}$. The definition of the binarization process (14)-(15) implies that, for any $k \in G$, we have (see (52) in Theorem 25)

$$
\omega_{j}^{k} \geq c_{j l}, j l \in L
$$

and the construction of the sufficient-equivalent set of cut points (Definition 6) implies that there exists $k^{\prime} \in G$ such that

$$
\omega_{j}^{k^{\prime}}=c_{j l}, \quad j l \in L .
$$

Constraints (33) and (34) ensure that exactly one term $\hat{w}_{j l} c_{j l}$ in the left-hand side of each constraint (61) is non-zero and equal to $c_{j l}, j l \in L$, and thus $y_{j}=c_{j l}, j l \in L$ in (61). This allows the rewriting of (65) as

$$
\omega_{j}^{k^{\prime}}=c_{j l}=\sum_{i=1}^{n_{j}} \hat{w}_{j l} c_{j l}=y_{j}, j l \in L .
$$

Since $k^{\prime} \in G \subseteq \bar{\Omega}_{B}^{+}$, (56) and (57) hold, which, along with (66), implies that

$$
\sum_{j \in J} s_{i j} y_{j} x_{j} \leq d_{i}, i=1, \ldots, r \quad \Rightarrow \quad \mathbb{P}(T x \leq d) \geq p .
$$

(ii) Any solution feasible for SRTM is feasible for QDE. For any $k^{\prime \prime}$ such that $\mathbb{P}\left(\xi \leq \omega^{k^{\prime \prime}}\right) \geq p$, the definition of the sufficient-equivalent set of cut points implies that there exists $k^{\prime} \in \bar{\Omega}_{B}^{+}$such that $\omega^{k^{\prime}} \leq$ $\omega^{k^{\prime \prime}}$ and $\omega_{j}^{k^{\prime}}=\underset{l=1, \ldots, n_{j}}{\bigvee} c_{j l}, j \in J$.

Let $l_{j}^{*}=\underset{l}{\operatorname{argmax}} \omega_{j}^{k^{\prime}}=c_{j l}, j \in J$. What is left to prove is that the vector $w^{\prime}$

$$
w_{j l}^{\prime}=\left\{\begin{array}{ll}
1 & \text { if } l=l_{j}^{*} \\
0 & \text { otherwise }
\end{array}, j \in J\right.
$$


is feasible for QDE, i.e., that $w^{\prime} \in\{w$ : (32), (33), (34), (61) $\}$. It is evident that $w^{\prime}$ is feasible for (33), (34) and (61). By construction (68), $w^{\prime}$ is feasible for (32) if

$$
\sum_{j \in J} \sum_{l=1}^{n_{j}} w_{j l}^{\prime} \beta_{j l}^{k}=\sum_{j \in J} w_{j l_{j}^{*}}^{\prime} \beta_{j l_{j}^{*}}^{k}=\sum_{j \in J} \beta_{j l_{j}^{*}}^{k} \leq|J|-1, k \in \bar{\Omega}_{B}^{-} .
$$

The feasibility of the above constraints is ensured if $\beta_{j l_{j}^{*}}^{k}=0$ for at least one $j, \forall k \in \bar{\Omega}_{B}^{-}$.

Note that, for any $k^{\prime} \in \bar{\Omega}_{B}^{+}$, there is no $k \in \bar{\Omega}_{B}^{-}$such that $\beta^{k} \geq \beta^{k^{\prime}}$ (see Definition 19 of a sufficientequivalent set of cut points). This implies that

$$
\left(\beta_{j 1}^{k}, \ldots, \beta_{j n_{j}}^{k}\right)<\left(\beta_{j 1}^{k^{\prime}}, \ldots, \beta_{j n_{j}}^{k^{\prime}}\right) \text { for at least one } j, \forall k \in \bar{\Omega}_{B}^{-} \text {. }
$$

Let $h \in J$ be the coordinate such that $\left(\beta_{h 1}^{k}, \ldots, \beta_{h n_{h}}^{k}\right)<\left(\beta_{h 1}^{k^{\prime}}, \ldots, \beta_{h n_{h}}^{k^{\prime}}\right)$ for an arbitrary $k \in \bar{\Omega}_{B}^{-}$. Since $l_{h}^{*}=\underset{l}{\operatorname{argmax}} \beta_{h l}^{k^{\prime}}=1$, we have for any $l>l_{h}^{*}$ that $\beta_{h l}^{k}=\beta_{h l}^{k^{\prime}}=0$. Thus, the vectors $\beta^{k^{\prime}}$ and $\beta^{k}$ differ only in terms of the $l_{h}^{*}$ first components, and we have

$$
\left(\beta_{h 1}^{k}, \ldots, \beta_{h l_{h}^{*}}^{k}\right)<\left(\beta_{h 1}^{k^{\prime}}, \ldots, \beta_{h l_{h}^{*}}^{k^{\prime}}\right)
$$

The regularization property (16)-(17) indicates that this relationship can only be true if $\beta_{h l_{h}^{*}}^{k}=0<\beta_{h l_{h}^{*}}^{k^{\prime}}=$ 1. This shows that, for any $k \in \bar{\Omega}_{B}^{-}, \beta_{j l_{j}^{*}}^{k}=0$ for at least one $j$, which results in $\sum_{j \in J} \beta_{j l_{j}^{*}}^{k}$ to be bounded from above by $(|J|-1)$ for each $k \in \bar{\Omega}_{B}^{-}$and implies that $w^{\prime}$ is feasible for (69) and thus (32).

Note that (63) is a strengthening of $y \in \mathbb{R}_{+}$and follows immediately from (33), (34), and (61). Problem QDE is a mixed-integer nonlinear programming (MINLP) problem which is NP-hard. It includes $n$ mixed-integer bilinear terms $x_{j} w_{j l}$ and its continuous relaxation is non-convex. In our example, the problem QDE reads:

$\max 2 x_{1}+x_{2}+x_{3}$

subject to (44) - (49)

$$
\begin{aligned}
& 7 w_{11}=y_{1} ; \quad 5 w_{21}+6 w_{22}+10 w_{23}=y_{2} ; \quad 3 w_{31}+4 w_{32}+5 w_{33}=y_{3} \\
& 2 y_{1} x_{1}+3 y_{2} x_{2} \leq 25 \\
& 3 y_{1} x_{1}+6 y_{3} x_{3} \leq 32 \\
& 5 \leq y_{2} \leq 10 ; \quad 3 \leq y_{3} \leq 5 \\
& x_{1}, x_{2}, x_{3} \geq 0
\end{aligned}
$$

\subsubsection{Mixed-Integer Linear Programming Formulation}

Instead of attempting to solve the QDE problem directly, we shall linearize the $|J|$ products $x_{j}\left(\sum_{l=1}^{n_{j}} w_{j l} c_{j l}\right)$ of continuous by binary variables (see, e.g., $[1,10]$ ). 
Theorem 28 The bilinear set

$$
S_{B}=\left\{(x, w) \in \mathbb{R}_{+}^{|J|} \times\{0,1\}^{n}: \sum_{j \in J} s_{i j} x_{j}\left(\sum_{l=1}^{n_{j}} w_{j l} c_{j l}\right) \leq d_{i} ;(33)\right\}
$$

is equivalent to the linear set

$S_{L}=\left\{(x, w, z) \in \mathbb{R}_{+}^{2|J|} \times\{0,1\}^{n} \times \mathbb{R}_{+}^{|J|}: \sum_{j \in J} s_{i j} z_{j} \leq d_{i} ; z_{j} \geq c_{j l} x_{j}-\left(1-w_{j l}\right) M_{j l}, j \in J, l=1, \ldots, n_{j} ;(33)\right\}$

where

$$
M_{j l}=\frac{d_{i} c_{j l}}{s_{i j} c_{j 1}}, j \in J, l=1, \ldots, n_{j} .
$$

Proof. First, we show that $M_{j l}$ is an upper bound on the value that $c_{j l} x_{j}$ can take. Consider an arbitrary $j \in J$. We derive now the upper bound for the corresponding variable $x_{j}$ induced by the constraint

$$
\sum_{j \in J} s_{i j} x_{j}\left(\sum_{l=1}^{n_{j}} w_{j l} c_{j l}\right) \leq d_{i}
$$

in $S_{B}$ (70). The value that $x_{j}$ can take is maximal if $x_{j^{\prime}}=0, \forall j^{\prime} \neq j$; in which case, (73) becomes:

$$
x_{j}\left(\sum_{l=1}^{n_{j}} w_{j l} c_{j l}\right) \leq \frac{d_{i}}{s_{i j}} .
$$

Since the cut points $c_{j l}$ are arranged in increasing order and (33) requires that one $w_{j l}$ is equal to 1 , while all the other $w_{j l}$ are equal to 0 , the smallest value that the expression $\left(\sum_{l=1}^{n_{j}} w_{j l} c_{j l}\right)$ can take is equal to $c_{j 1}$ when $w_{j 1}=1$. Therefore, we have

$$
x_{j} \leq \frac{d_{i}}{s_{i j} c_{j 1}} .
$$

and we obtain the upper bound $M_{j l}=\frac{d_{i} c_{j l}}{s_{i j} c_{j 1}}$ for the terms $c_{j l} x_{j}, j \in J, l=1, \ldots, n_{j}$.

We shall now prove that the feasible sets defined by $S_{B}$ and $S_{L}$ are equivalent and that the substitution of $z_{j}$ for $x_{j}\left(\sum_{l=1}^{n_{j}} w_{j l} c_{j l}\right)$ in (73) is valid. In order to do so, we show that:

1. The linearized constraints

$$
z_{j} \geq c_{j l} x_{j}-\left(1-w_{j l}\right) M_{j l}, j \in J, l=1, \ldots, n_{j},
$$

in $S_{L}$ (71), with $z_{j} \geq 0, j \in J$, imply that

$$
z_{j} \geq x_{j}\left(\sum_{l=1}^{n_{j}} w_{j l} c_{j l}\right) .
$$

The set partitioning constraints (33) imply that exactly one $w_{j l}, l=1, \ldots n_{j}$ is equal to 1 for each $j \in J$. Therefore, (75) can be rewritten as:

$$
z_{j} \geq x_{j} w_{j l} c_{j l}, j \in J, l=1, \ldots, n_{j}
$$


Consider an arbitrary component $j \in J$ and let $l_{j}^{\prime}$ be such that $w_{j l_{j}^{\prime}}=1, w_{j l}=0, \forall l \neq l_{j}^{\prime}$. Then, we have

$$
z_{j} \geq x_{j} w_{j l} c_{j l}=\left\{\begin{array}{l}
x_{j} c_{j l_{j}^{\prime}}, l=l_{j}^{\prime}, j \in J \\
0, l \neq l_{j}^{\prime}, j \in J
\end{array}\right.
$$

Observe that for $l=l_{j}^{\prime}$ the inequality (74) reads $z_{j} \geq c_{j l_{j}^{\prime}} x_{j}$ and is the same as in (77). Since $z_{j} \geq$ $0, j \in J$, we have shown that the constraints (74) imply (75). Hence, if we replace $x_{j}\left(\sum_{l=1}^{n_{j}} w_{j l} c_{j l}\right)$ by $z_{j}$ in (73), we have

$$
\sum_{j \in J} s_{i j} z_{j} \leq d_{i} \Rightarrow \sum_{j \in J} s_{i j} x_{j}\left(\sum_{l=1}^{n_{j}} w_{j l} c_{j l}\right) \leq d_{i} .
$$

2. The linearized constraints do not cut any solution feasible of $S_{B}$.

For $l=l_{j}^{\prime}$, the inequality (74) requires $z_{j}$ to be at least equal to $c_{j l_{j}^{\prime}} x_{j}$ and is equivalent to (77). Consider now the other indices $l \neq l_{j}^{\prime}$ for which $w_{j l}=0$. The corresponding $(j, l)$-constraints $(74)$ in $S_{L}$ imply that

$$
z_{j} \geq c_{j l} x_{j}-M_{j l}, \forall j \neq l_{j}^{\prime} .
$$

Since each parameter $M_{j l}$ limits from above $c_{j l} x_{j}$, we have: $c_{j l} x_{j}-M_{j l} \leq 0$ and $z_{j} \geq 0$. It is thus evident that the $(j, l)$-constraints $(74)\left(l \neq l_{j}^{\prime}\right)$ are not binding (since $\left.z_{j} \geq 0\right)$ and do not cut any feasible solution of $S_{B}$.

A direct consequence of Theorem 28 is that the bilinear integer problem QDE can be equivalently reformulated as the MIP problem MIP1. Lemma 29 follows.

Lemma 29 The probabilistically constrained stochastic programming problem SRTM can be equivalently reformulated as the MIP problem MIP1:

$$
\begin{array}{rll}
\text { MIP1 : max } & q^{\top} x & \\
\text { subject to } & (2) ;(4) ;(32) ;(33) ;(34) & \\
& \sum_{j \in J} s_{i j} z_{j} \leq d_{i}, & i=1, \ldots, r \\
z_{j} \geq c_{j l} x_{j}-\left(1-w_{j l}\right) \bar{M}_{j l}, & j \in J, l=1, \ldots, n_{j} \\
z_{j} \geq 0, & j \in J,
\end{array}
$$

where $\bar{M}_{j l}=\max _{i: s_{i j} \neq 0} \frac{d_{i} c_{j l}}{s_{i j} c_{j 1}}, j \in J, l=1, \ldots, n_{j}$. 
In our example, MIP1 is given by:

$$
\max 2 x_{1}+x_{2}+x_{3}
$$

subject to (44) - (49)

$$
\begin{aligned}
& 2 z_{1}+3 z_{2} \leq 25 \\
& 3 z_{1}+6 z_{3} \leq 32 \\
& z_{1} \geq 7 x_{1}-\left(1-w_{11}\right) \cdot \frac{25}{2} \\
& z_{2} \geq 5 x_{2}-\left(1-w_{21}\right) \cdot \frac{25}{3} ; \quad z_{2} \geq 6 x_{2}-\left(1-w_{22}\right) \cdot 15 ; \quad z_{2} \geq 10 x_{2}-\left(1-w_{23}\right) \cdot 4 \\
& z_{3} \geq 3 x_{3}-\left(1-w_{31}\right) \cdot \frac{32}{6} ; \quad z_{3} \geq 4 x_{3}-\left(1-w_{32}\right) \cdot \frac{128}{18} ; \quad z_{3} \geq 5 x_{3}-\left(1-w_{33}\right) \cdot \frac{160}{18} \\
& x_{j}, z_{j} \geq 0, j=1,2,3
\end{aligned}
$$

\subsubsection{Strengthened Mixed-Integer Linear Programming Formulation}

We shall now strengthen the linearized formulation MIP1 and derive valid inequalities. Consider the set

$$
G_{j}=\left\{(x, z, w) \in \mathbb{R}_{+}^{2} \times\{0,1\}^{n_{j}}: z_{j} \geq c_{j l} x_{j}-\left(1-w_{j l}\right) \bar{M}_{j l}, l=1, \ldots, n_{j} ; \sum_{j=1}^{n_{j}} w_{j l}=1\right\} .
$$

Theorem 30 The inequalities

$$
\begin{gathered}
z_{j} \geq c_{j l} x_{j}-\left(1-v_{j l}\right) \bar{M}_{j l}, l=1, \ldots, n_{j}, \\
v_{j l}=\sum_{r=l}^{n_{j}} w_{j r}, l=1, \ldots, n_{j},
\end{gathered}
$$

are valid for $G_{j}$ and are tighter than

$$
z_{j} \geq c_{j l} x_{j}-\left(1-w_{j l}\right) \bar{M}_{j l}, l=l^{\prime}, \ldots, n_{j}
$$

Proof. Let $w$ be feasible for the continuous relaxation of the set $G_{j}$ and let $l^{\prime}=\underset{l}{\operatorname{argmax}} w_{j l}>0$.

For $l \geq l^{\prime}, v_{j l}=w_{j l}$ and the corresponding inequalities (85) and (87) in $G_{j}$ are identical.

We show now that the inequalities (85) are tighter than (87) for $l^{\prime}>l$. For $l<l^{\prime}\left(l^{\prime}>1\right)$, we have $v_{j l} \geq w_{j l}$ from (86), which successively implies that

$$
\left(1-w_{j l}\right) \bar{M}_{j l} \geq\left(1-v_{j l}\right) \bar{M}_{j l} \quad \Rightarrow \quad c_{j l} x_{j}-\left(1-v_{j l}\right) \bar{M}_{j l} \geq c_{j l} x_{j}-\left(1-w_{j l}\right) \bar{M}_{j l} .
$$

Thus, $z_{j}$ is limited from below by $c_{j l} x_{j}-\left(1-v_{j l}\right) \bar{M}_{j l}$ in (85) which is larger than or equal to the lower bound $c_{j l} x_{j}-\left(1-w_{j l}\right) \bar{M}_{j l}$ on $z_{j}$ defined by (87). Further, from (86) and the fact that $l^{\prime}=\operatorname{argmax} w_{j l}>0$, it follows that $v_{j l}>w_{j l}, l=1, \ldots, l^{\prime}-1$. Hence, $\left(l^{\prime}-1\right)$ of the inequalities (88) are strict. This shows that the inequalities (85) $\left(l<l^{\prime}\right)$ are tighter than (87). 
We show now that the inequalities (85) do not cut any feasible solution of $G_{J}$ and are thus valid. Recall that $l^{\prime}=\operatorname{argmax} w_{j l}>0$. From any fractional solution $w$, we can easily derive a integral solution for (85) and (87) by setting: $v_{j l}=1, l=1, \ldots, l^{\prime}, v_{j l}=0, l>l^{\prime}, w_{j l^{\prime}}=1$, and $w_{j l}=0, l \neq l^{\prime}$. The inequalities (85) read

$$
z_{j} \geq c_{j l} x_{j}, l=1, \ldots, l^{\prime} .
$$

Observe first that the inequality (87) corresponding to the component $l^{\prime}$ requires

$$
z_{j} \geq c_{j l^{\prime}} x_{j}
$$

Note further that, since the cut points are sorted in ascending order (15), we have that

$$
c_{j l^{\prime}} x_{j} \geq c_{j l} x_{j}, l=l^{\prime}, \ldots, n_{j} .
$$

The above relationships (90) and (91) imply that the constraints (89) do not cut any feasible solution for $G_{j}$ and are thus valid.

Observe that, by construction (86), we have $v_{j 1}=1$ and $z_{j} \geq c_{j 1} x_{j}$.

Lemma 31 is a direct consequence of Theorem (30)

Lemma 31 Problems MIP1 and MIP2 are equivalent and therefore problem SRTM can be equivalently reformulated as the MIP problem MIP2:

MIP2 : $\max$

$$
q^{\top} x
$$

subject to

(2); (4); (32); (33); (34); (79); (81)

$$
\begin{array}{cl}
z_{j} \geq c_{j l} x_{j}-\left(1-v_{j l}\right) \bar{M}_{j l}, & j \in J, l=1, \ldots, n_{j} \\
v_{j l}=\sum_{r=l}^{n_{j}} w_{j r}, & j \in J, l=1, \ldots, n_{j}
\end{array}
$$

In our example, MIP2 reads:

$$
\begin{array}{rlrl}
\max & 2 x_{1}+x_{2}+x_{3} \\
\text { subject to }(44)-(49) ;(82)-(83) & & \\
z_{1} & \geq 7 x_{1}-\left(1-v_{11}\right) \cdot \frac{25}{2} & & \\
z_{2} & \geq 5 x_{2}-\left(1-v_{21}\right) \cdot \frac{25}{3} ; \quad z_{2} \geq 6 x_{2}-\left(1-v_{22}\right) \cdot 15 ; \quad z_{2} \geq 10 x_{2}-\left(1-v_{23}\right) \cdot 4 \\
z_{3} & \geq 3 x_{3}-\left(1-v_{31}\right) \cdot \frac{32}{6} & & \\
z_{3} & \geq 4 x_{3}-\left(1-v_{32}\right) \cdot \frac{128}{18} & & \\
z_{3} & \geq 5 x_{3}-\left(1-v_{33}\right) \cdot \frac{160}{18} & & \\
v_{11} & =w_{11} \\
v_{21} & =w_{21}+w_{22}+w_{23} ; & v_{22}=w_{22}+w_{23} ; & v_{33}=w_{33} \\
v_{31} & =w_{31}+w_{32}+w_{33} ; & v_{32}=w_{32}+w_{33} ; & \\
x_{j}, z_{j} \geq 0, j=1,2,3 & &
\end{array}
$$


The optimal solution $\left(x^{*}, w^{*}\right)=(\underbrace{1.52,0.52,1.19}_{x^{*}} ; \underbrace{1,1 / 3,2 / 3,0,2 / 3,0,1 / 3,1 / 3,1 / 3}_{w^{*}})$ of the continuous relaxation of problem MIP1 violates the constraints (94), (95), and (96) in the continuous relaxation of problem MIP2.

\section{Numerical Evaluation}

\subsection{Test Laboratory}

In this section, we test the contribution of the solution framework for the numerical solution of optimization problems including multi-row probabilistic constraints with random technology matrix. The tests are conducted on instances of a multi-period capital rationing problem in which uncertainty concerns the value of each possible project, the cash outflows associated with each of them, and the capital available at each period in the planning horizon. Random variables are in both the technology matrix and the right-hand sides of the probabilistic constraint.

The capital rationing process consists in the allocation of a limited budget to profitable projects. A survey considering Fortune 500 firms indicates that more than $60 \%$ of them place a limit on the internal capital available for investment plans [21]. We refer to [16] for a review of the mathematical programming approaches used for capital rationing purposes. The problem of rationing capital under uncertainty was first studied by Lorie and Savage [20] who describe the challenges of capital rationing problems involving multi-period projects that generate inflows and outflows dispersed across their lifetimes. The first chance-constrained formulation for capital rationing was proposed by Naslund [23] and maximizes the expected value of the adopted projects across a multi-period horizon, plus the amount of money lent, minus the amount of money borrowed. The model includes several individual chance constraints requiring that the probability of having a budget deficit in each period is kept low. Sarper [31] rejects the use of the normal distribution for characterizing the uncertain cash flows and supports the use of uniformly distributed variables. Gurgur and Luxhoj [11] assume that cash flows and available budgets are asymmetrically distributed random variables and follow a Weibull distribution. Most recently, Beraldi et al. [4] propose a formulation with joint probabilistic constraints in which random variables are finitely distributed. They propose a specialized branch-and-bound algorithm and evaluate the impact of introducing risk measures.

Let $r$ denote the number of periods in the horizon and $J$ be the set of possible projects. The binary decision variables $x_{j}(100)$ define whether project $j$ is selected (i.e., $x_{j}=1$ ) or not. The random variable $\xi_{i j}, i=1, \ldots, r, j \in J$ represents the cash outflow due to project $j$ in period $i$, while $\zeta_{i}, i=1, \ldots, r$ denotes the random budget that will be available in period $i$. We denote by $V_{j}$ the random value of project $j$. The initial cash outflows and the currently available budget are known and denoted by $a_{0 j}, j \in J$ and $b_{0}$. The formulation reads: 


$$
\begin{array}{cc}
\max & \sum_{j \in J} \mathbb{E}\left[V_{j}\right] \cdot x_{j} \\
\text { subject to } & \sum_{j \in J} a_{0 j} \cdot x_{j} \leq b_{0} \\
& \mathbb{P}\left(\sum_{j \in J} \xi_{i j} \cdot x_{j} \leq \zeta_{i}, i=1, \ldots, r\right) \geq p \\
x \in\{0,1\}^{|J|}
\end{array}
$$

The objective function (97) maximizes the expected value of the selected projects. Constraint (98) ensures that the initial cash outflows do not exceed the current budget. Constraint (99) requires that the cash outflows of the selected projects do not create a budget shortfall at any of the periods of the horizon with a probability at least equal to $p$.

The "aggregated" random matrix $T \in \mathbb{R}_{+}^{r \times(1+|J| r)}$ in SRTM includes the random cash outflows $\xi_{i j}$ and available budgets $\zeta_{i}$. Each row $T_{i}$ includes up to $(|J|+1)$ non-zero components:

$$
T_{i}=\left[0, \ldots, 0, \xi_{i 1}, \xi_{i 2}, \ldots, \xi_{i|J|}, 0, \ldots, 0,-\zeta_{i}, 0, \ldots, 0\right], i=1, \ldots, r .
$$

The first $(r \cdot|J|)$ components of $T_{i}$ are the cash flows incurred by each project $j$ at the successive periods $i$, while the $r$ last components of $T_{i}$ are the budgets that will be available in the future periods $i$.

Let's omit the zero-components in (101) and rewrite the vector $T_{i}$ of the random technology matrix $T$ accordingly: $T_{i}=\left[\xi_{i 1}, \xi_{i 2}, \ldots, \xi_{i l},-\zeta_{i}\right]$. We denote by $c_{j 1}, \ldots, c_{j n_{j}}, j \in J$ the cut points associated with the random variable $\xi_{i j}$ and by $c_{01}, \ldots, c_{0 n_{0}}$ the cut points associated with $\zeta_{i}$.

Owing to the structure of the capital rationing problem, namely the stochasticity of the right-hand side $d_{i}$, and $s_{i j}=1$, the set $S_{B}$ (70) corresponding to the $i^{\text {th }}$ inequality in (98) can be rewritten as

$$
S_{B}^{C R}=\left\{(x, w) \in\{0,1\}^{|J|+n+n_{0}}: \sum_{j \in J} x_{j}\left(\sum_{l=1}^{n_{j}} w_{j l} c_{j l}\right) \leq \sum_{l=1}^{n_{0}} w_{0 l} c_{0 l} ;(33)\right\}
$$

It is straightforward to see that the set

$$
S_{L}^{C R}=\left\{(x, w, z) \in\{0,1\}^{|J|+n+n_{0}} \times \mathbb{R}_{+}^{|J|}: \sum_{j \in J} s_{i j} z_{j} \leq \sum_{l=1}^{n_{0}} w_{0 l} c_{0 l} ; z_{j} \geq c_{j l} x_{j}-\left(1-w_{j l}\right) M_{j l}, \forall j, l ;(33)\right\}
$$

with $M_{j l}=\frac{c_{01} c_{j l}}{c_{j 1}}, j \in J, l=1, \ldots, n_{j}$ is equivalent to $S_{B}^{C R}$.

In order to find the tightest possible continuous relaxation of $S_{B}^{C R}$, the constants $M_{j l}, j \in J, l=1, \ldots, n_{j}$ should be set to the largest values that yet ensure the validity of the formulation. Taking into account the specifics of the capital rationing problem, Proposition 32 provides the largest values that can be assigned to the parameters $M_{j l}$.

Proposition 32 For every inequality $z_{j} \geq c_{j l} x_{j}-\left(1-w_{j l}\right) M_{j l}, j \in J, l=1, \ldots, n_{j}$ in $S_{L}^{C R}$,

$$
M_{j l}=\frac{d_{i} c_{j l}}{s_{i j} c_{j 1}}
$$

is the largest value that $M_{j l}$ can take to ensure the equivalence between $S_{L}$ and $S_{B}^{C R}$. 
Proof. The equivalence between $S_{L}^{C R}$ and $S_{B}^{C R}$ is based on the substitution of $z_{j}$ for $\sum_{j \in J} x_{j}\left(\sum_{l=1}^{n_{j}} w_{j l} c_{j l}\right)$, the incorporation of the set of constraints (99), and the assignment of acceptable values to the constants $M_{j l}$. Finding the largest valid value for each $M_{j l}$ can be represented as the optimization problem:

$$
\begin{gathered}
\max M_{j l} \\
\text { subject to } c_{j l} x_{j}-\left(1-w_{j l}\right) M_{j l} \geq x_{j}\left(\sum_{l=1}^{n_{j}} w_{j l} c_{j l}\right), \quad j \in J, l=1, \ldots, n_{j}
\end{gathered}
$$

The following cases can be considered for each $j$ :

1. If $x_{j}=0$, it is obvious that the value of $M_{j l}$ is unrestricted;

2. If $x_{j} \neq 0$ and thus equal to 1 :

- Consider the component $l^{\prime}$ for which $w_{j l^{\prime}}=1$. The corresponding $l^{\prime}$-inequality in (103) reads after substitution: $c_{j l^{\prime}} \geq c_{j l^{\prime}}$ and leaves the value of $M_{j l}$ unrestricted;

- Consider the components $l=1, \ldots, n_{j}, l \neq l^{\prime}$ for which $w_{j l}=0$. The $l$ corresponding inequalities in (103) reads after substitution:

$$
c_{j l}-M_{j l} \geq \sum_{l=1}^{n_{j}} w_{j l} c_{j l}=c_{j l^{\prime}} .
$$

Constraint (33) in $S_{L}^{C R}$ and $S_{B}^{C R}$ imply that exactly one $w_{j l}$ is equal to 1 . This, along with the ascending order (15) in which the cut points are sorted, imply that: $\sum_{l=1}^{n_{j}} w_{j l} c_{j l}=c_{j l^{\prime}} \geq c_{j 1}$. Therefore, we replace the right-hand side in (104) by its lower bound to find the largest valid for $M_{j l}$ and obtain: $c_{j l}-M_{j l} \geq c_{j 1}$. Clearly, the largest valid value for $M_{i j}$ is $c_{j l}-c_{1 l}$, which was set out to prove.

To generate the problem instances, we use the approach described by Beraldi et al. [4] and decompose a planning horizon into a finite number of periods. The budget available is known for the first period, while it is stochastic for the next ones. We consider a number of potential projects ranging between 10 and 20. Such numbers of projects are typical for the capital rationing problem, as the project selection is typically preceded by a screening phase in which homogeneous groups of projects are defined and are later on subjected to a joint evaluation [7]. The project outflows $\xi_{i j}$ and project values $V_{j}$ have been randomly generated from a uniform distribution (see $[4,31]$ ) defined on $[300,600]$ and $[10,1000]$, respectively. We also generate the available budgets from a uniform distribution defined on $\left[0.2 \cdot C_{i}, C_{i}\right]$, where $C_{i}$ is the average (over all potential projects) cash outflow at period $i$. We create 36 types of problem instances characterized by the tuple $(|\Omega|, r,|J|, p)$. The instances differ in terms of the number of realizations $(|\Omega|=10000,15000)$, the number of periods $(r=1,2,3)$ in the planning horizon, the number of considered projects $(|J|=10,20)$, and the enforced probability level ( $p=0.9,0.925,0.95)$. For each instance type, we generate four problem instances and solve the 144 problem instances with the four 
algorithms described in Section 5.2. The binarization process employed for deriving the proposed MIP formulations is implemented in Matlab. The AMPL modeling language is used to formulate the mathematical programming problems. The mixed-integer programming formulations are solved with the Cplex 12.4 solver and the mixed-integer nonlinear programming ones (with nonconvex continuous relaxations) are solved with the Couenne solver. Each problem instance is solved on a 64-bit Dell Precision T5400 Workstation with Quad Core Xeon Processor X5460 3.16GHz CPU, and 4X2GB of RAM. The next subsection analyzes the computational tractability of the several equivalent problem formulations proposed in 4.2 .

\subsection{Comparative Analysis of Equivalent Reformulations and Algorithmic Techniques}

As mentioned above, we have created 144 problem instances. For each of them, we

- use the MINLP solver Couenne [2] to solve the bilinear integer problem QDE with nonconvex continuous relaxation;

- use Cplex 12.4's standard branch-and-bound algorithm to solve the MIP problem MIP1;

- use Cplex 12.4's standard branch-and-bound algorithm supplemented by the valid inequalities (85) to solve the MIP problem MIP2;

- use a new branch-and-cut algorithm in which, at each node of the tree, the valid inequalities (85) violated by the optimal solution of the incumbent continuous relaxation are introduced in the formulation of the problem.

The above four methods will be referred to with the acronyms QDE, MIP1, MIP2-B\&B, and MIP2$\mathbf{B} \& \mathbf{C}$ to analyze the computational results associated with each of them. The first five columns in Table 3 describe the problem instances. Column 1 (resp., 2, 3, and 4 5) indicates the number of scenarios (resp., periods, projects, and random variables), while the fifth column gives the enforced reliability level. The last four columns provide the average computational time with each algorithm for each of the 36 types of problem instances. The expressions " $>3600 "$ means that the problem instance could not be solved to optimality within one hour of CPU time.

Not surprisingly, the MINLP formulation QDE takes much more time to solve than its three MIP counterparts. The QDE problems are solved with the MINLP solver Couenne and only four instance types can be solved to optimality within one hour of computing time. The other three algorithms MIP1, MIP2-B\&B, and MIP2-B\&C solve to optimality all the 144 problem instances. To distinguish among these three algorithms, we focus on the 10 problem instance types (i.e., 40 problem instances) which require an average computing time of at least 5 seconds. The MIP2-B\&B (resp., MIP2-B\&C and MIP1) algorithm is the fastest for 7 instances types (resp., 2 and 1). For the methods MIP1, MIP2-B\&B, and MIP2-B\&C, Figures 1 and 2 display the average computational time for the 40 problem instances with computational time above 5 seconds and the 12 problem instances with computational time above 20 
Table 3: Average Computational Times for each Algorithm

\begin{tabular}{c|c|c|c|c||c|c|c|c}
\hline$|\Omega|$ & $r$ & $|J|$ & $r \cdot(|J|+1)$ & $p$ & QDE & MIP1 & MIP2-B\&B & MIP2-B\&C \\
\hline 10000 & 1 & 10 & 11 & 0.95 & 5.80 & 0.02 & 0.02 & 0.02 \\
10000 & 1 & 10 & 11 & 0.925 & 20.31 & 0.03 & 0.02 & 0.03 \\
10000 & 1 & 10 & 11 & 0.9 & 364.27 & 0.05 & 0.06 & 0.08 \\
\hline 10000 & 1 & 20 & 21 & 0.95 & $>3600$ & 0.03 & 0.02 & 0.02 \\
10000 & 1 & 20 & 21 & 0.925 & $>3600$ & 0.25 & 0.25 & 0.25 \\
10000 & 1 & 20 & 21 & 0.9 & $>3600$ & 6.71 & 5.94 & 6.41 \\
\hline 10000 & 2 & 10 & 22 & 0.95 & 2487.86 & 0.03 & 0.03 & 0.02 \\
10000 & 2 & 10 & 22 & 0.925 & 2614.00 & 0.19 & 0.22 & 0.22 \\
10000 & 2 & 10 & 22 & 0.9 & $>3600$ & 5.65 & 5.63 & 5.80 \\
\hline 10000 & 2 & 20 & 42 & 0.95 & $>3600$ & 0.09 & 0.09 & 0.11 \\
10000 & 2 & 20 & 42 & 0.925 & $>3600$ & 1.30 & 0.58 & 0.53 \\
10000 & 2 & 20 & 42 & 0.9 & $>3600$ & 51.57 & 33.10 & 45.05 \\
\hline 10000 & 3 & 10 & 33 & 0.95 & $>3600$ & 0.03 & 0.02 & 0.02 \\
10000 & 3 & 10 & 33 & 0.925 & $>3600$ & 0.36 & 0.30 & 0.28 \\
10000 & 3 & 10 & 33 & 0.9 & $>3600$ & 6.85 & 6.66 & 6.72 \\
\hline 10000 & 3 & 20 & 63 & 0.95 & $>3600$ & 0.17 & 0.19 & 0.20 \\
10000 & 3 & 20 & 63 & 0.925 & $>3600$ & 1.30 & 1.34 & 1.25 \\
10000 & 3 & 20 & 63 & 0.9 & $>3600$ & 117.59 & 87.10 & 101.67 \\
\hline 15000 & 1 & 10 & 11 & 0.95 & 1.62 & 0.02 & 0.02 & 0.02 \\
15000 & 1 & 10 & 11 & 0.925 & 1.65 & 0.03 & 0.02 & 0.03 \\
15000 & 1 & 10 & 11 & 0.9 & 42.42 & 0.06 & 0.08 & 0.08 \\
\hline 15000 & 1 & 20 & 21 & 0.95 & $>3600$ & 0.03 & 0.03 & 0.02 \\
15000 & 1 & 20 & 21 & 0.925 & $>3600$ & 0.23 & 0.25 & 0.25 \\
15000 & 1 & 20 & 21 & 0.9 & $>3600$ & 6.43 & 6.96 & 6.93 \\
\hline 15000 & 2 & 10 & 22 & 0.95 & 1514.00 & 0.03 & 0.03 & 0.02 \\
15000 & 2 & 10 & 22 & 0.925 & 1452.90 & 0.23 & 0.22 & 0.25 \\
15000 & 2 & 10 & 22 & 0.9 & $>3600$ & 6.18 & 6.68 & 6.05 \\
\hline 15000 & 2 & 20 & 42 & 0.95 & $>3600$ & 0.08 & 0.06 & 0.08 \\
15000 & 2 & 20 & 42 & 0.925 & $>3600$ & 0.58 & 0.56 & 0.53 \\
15000 & 2 & 20 & 42 & 0.9 & $>3600$ & 57.19 & 25.74 & 52.12 \\
\hline 15000 & 3 & 10 & 33 & 0.95 & $>3600$ & 0.02 & 0.03 & 0.03 \\
15000 & 3 & 10 & 33 & 0.925 & $>3600$ & 0.36 & 0.31 & 0.30 \\
15000 & 3 & 10 & 33 & 0.9 & $>3600$ & 7.27 & 6.89 & 6.88 \\
\hline 15000 & 3 & 20 & 63 & 0.95 & $>3600$ & 0.20 & 0.28 & 0.33 \\
15000 & 3 & 20 & 63 & 0.925 & $>3600$ & 1.61 & 1.72 & 1.72 \\
15000 & 3 & 20 & 63 & 0.9 & $>3600$ & 95.48 & 66.71 & 80.81 \\
\hline & & & & & & &
\end{tabular}


seconds, respectively. For the instances requiring more than 5 (resp., 20) seconds of computing time, the MIP2-B\&B algorithm is on average more than 26\% and 43\% (resp. $31 \%$ and 51\%) faster than the MIP1 and MIP2-B\&C methods. The MIP2-B\&C method outpaces the MIP1 method by $13 \%$ (resp., 15\%) on the instances requiring more than 5 (resp., 20) seconds. The MIP2-B\&B and MIP2-B\&C methods which both use in some fashion the valid inequalities (85) are faster than the MIP1 method, which points out the benefits of the valid inequalities (85). Among the two, the branch-and-bound algorithm MIP2B\&B outperforms the branch-and-cut algorithm MIP2-B\&C for the instances considered in this study.

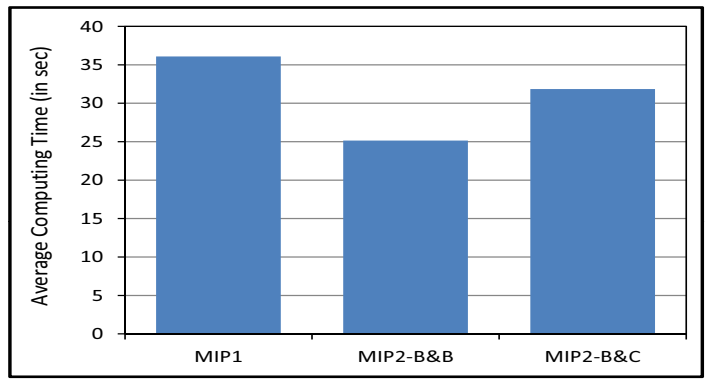

Figure 1: Average Computational Time for Instances Requiring more than 5 Seconds

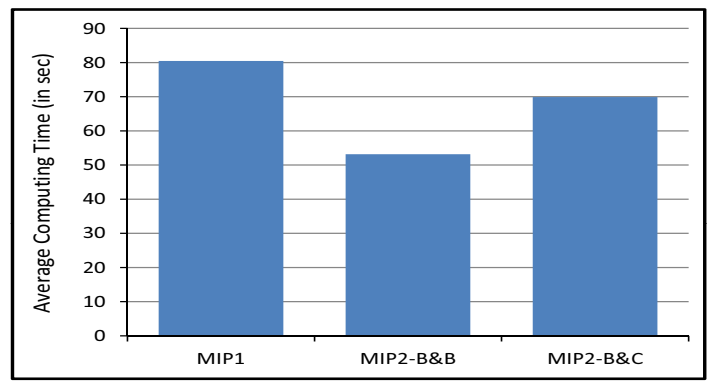

Figure 2: Average Computational Time for Instances Requiring more than 20 Seconds

It can be seen that the computing time does not increase with the number of scenarios used to represent uncertainty. This is highlighted by Figure 3 which displays the average computational time of the MIP1, MIP2-B\&B, and MIP2-B\&C algorithms for the two instance types $((|\Omega|, r,|J|, p)=$ $(10000,3,20,0.9)$ and $=(15000,3,20,0.9))$ that are the most time consuming and that only differ in the considered number of scenarios (10000 vs. 15000). It can be seen that the average solution time is smaller for the instances including 15000 scenarios than for those including 10000 scenarios.

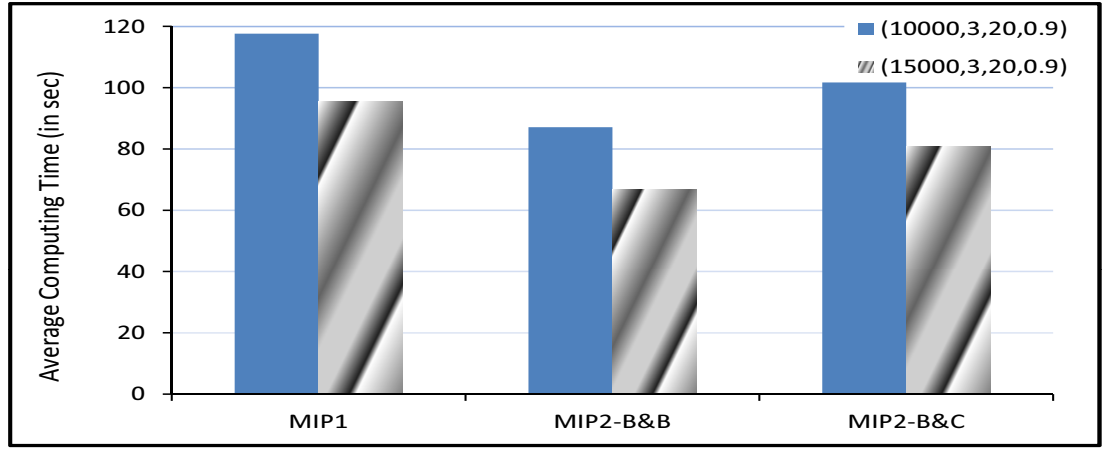

Figure 3: Average Computational Time as a Function of Number of Scenarios

Finally, we also notice that the computational time is a decreasing function of the enforced reliability level $p$. All the instances requiring more than 5 seconds of computing time have a reliability level of $90 \%$. As the value of $p$ decreases, the number of cut points needed to binarize the probability distribution and therefore the number of binary variables in MIP1 and MIP2 increases. For the instances under consideration, the number of constraints of type (32) increases fast for lower values of $p$. The computing time is also an increasing function of the size of the random vector. 


\section{Conclusion}

We develop a new modeling and exact solution method for stochastic programming problems that include a joint probabilistic constraint in which the multi-row random technology matrix is discretely distributed. We binarize the probability distribution of the random variables in such a way that we can extract a threshold partially defined Boolean function (pdBf) representing the probabilistic constraint. We then construct a tight threshold Boolean minorant for the pdBf. Any separating structure of the tight threshold Boolean minorant defines sufficient conditions for the satisfaction of the probabilistic constraint and takes the form of a system of linear constraints. We use the separating structure to derive three new deterministic formulations equivalent to the studied stochastic problem. We derive a set of strengthening valid inequalities for the reformulated problems. A crucial feature of the new integer formulations is that the number of integer variables does not depend on the number of scenarios used to represent uncertainty. The proposed reformulation method makes it possible to solve very efficiently probabilistically constrained stochastic programming problems with multi-row random technology matrix. The computational study, based on instances of the stochastic capital rationing problem, shows that the MIP reformulations are much easier and orders of magnitude faster to solve than the MINLP formulation. The method integrating the derived valid inequalities in a branch-and-bound algorithm performs best.

\section{References}

[1] Adams W.P., Sherali H.G. 1993. Mixed-Integer Bilinear Programming Problems. Mathematical Programming 59, 279-305.

[2] Belotti P. 2009. Couenne: A User's Manual. Technical Report, Lehigh University.

[3] Beraldi P., Bruni M.E. 2010. An Exact Approach for Solving Integer Problems Under Probabilistic Constraints with Random Technology Matrix. Annals of Operations Research 177, 127-137.

[4] Beraldi P., Bruni M.E., Violi A. 2012. Capital Rationing Problems under Uncertainty and Risk. Computational Optimization and Applications 51 (3), 1375-1396.

[5] Bonami P., Lejeune M.A. 2009. An Exact Solution Approach for Portfolio Optimization Problems Under Stochastic and Integer Constraints. Operations Research 57 (3), 650-670.

[6] Boros E., Hammer P.L., Ibaraki T., Kogan A. 1997. Logical Analysis of Numerical Data. Mathematical Programming 79, 163-190.

[7] Costa A., Paixao J.M.P. 2010. An Approximate Solution Approach for a Scenario-Based Capital Budgeting Model. Computational Management Science 7 (3), 337-353.

[8] Crama Y., Hammer P.L. 2011. Boolean Functions - Theory, Algorithms, and Applications. Cambridge Press University.

[9] Genz A., Bretz F. 2009. Computation of Multivariate Normal and $t$ Probabilities. Lecture Notes in Statistics 195, Dordrecht, Springer.

[10] Glover F. 1975. Improved Linear Integer Programming Formulations of Nonlinear Integer Problems. Management Science 22 (4), 455-460.

[11] Gurgur C. Z., Luxhoj J. T. 2003. Application of Chance-Constrained Programming to Capital Rationing Problems with Asymmetrically Distributed Cash Flows and Available Budgets. The Engineering Economist 48 (3), 241-258. 
[12] Henrion R. 2007. Structural Properties of Linear Probabilistic Constraints. Optimization 56, 425440.

[13] Henrion R., Strugarek C. 2008. Convexity of Chance Constraints with Independent Random Variables. Computational Optimization and Applications 41 (2), 263-276.

[14] Ibaraki T. 2011. Partially Defined Boolean Functions. In: Boolean Functions - Theory, Algorithms, and Applications. Eds: Crama Y., Hammer P.L. Cambridge Press University.

[15] Kataoka S. 1963. A Stochastic Programming Model. Econometrica 31 (12), 181-196.

[16] Khan A. 2008. Capital Budgeting Under Capital Rationing: An Analytical Overview of Optimization Models for Government. International Journal of Public Administration 31 (2), 168-194.

[17] Lejeune M.A. 2011. A VaR Black-Litterman Model for the Construction of Absolute Return Fundof-Funds. Quantitative Finance 11, 639-664.

[18] Lejeune M.A. 2012. Pattern-Based Modeling and Solution of Probabilistically Constrained Optimization Problems. Operations Research. In Press.

[19] Lejeune M.A. 2012. Pattern Definition of the p-Efficiency Concept. Annals of Operations Research. In Press.

[20] Lorie J.H., Savage L.J. 1955. Three Problems in Rationing Capital. The Journal of Business 28 (2), 229-239.

[21] Mukherjee T., Hingorani V. 1999. Capital-Rationing Decisions of Fortune 500 Firms: A Survey. Financial Practice and Education 1, 7-15.

[22] Muroga S. 1971. Threshold Logic and its Applications. Wiley-Interscience, New York, NY.

[23] Naslund B. 1966. A Model of Capital Budgeting under Risk. Journal of Business 39 (2), 257-271.

[24] Prékopa A. 1970. On Probabilistic Constrained Programming. Proceedings of the Princeton Symposium on Mathematical Programming. Princeton University Press, 113-138.

[25] Prékopa A. 1973. Contributions to the Theory of Stochastic Programming. Mathematical Programming 4, 202-221.

[26] Prékopa A. 1974. Programming under Probabilistic Constraints with a Random Technology Matrix. Mathematische Operationsforschung und Statistik 5, 109-116.

[27] Prékopa A. 1995. Stochastic Programming. Kluwer. Boston, MA.

[28] Prékopa A. 2003. Probabilistic Programming Models. Chapter 5 in: Stochastic Programming: Handbook in Operations Research and Management Science 10. Eds: Ruszczyński A., Shapiro A. Elsevier Science Ltd, 267-351.

[29] Prékopa A., Yoda K., Subasi M. 2011. Uniform Quasi-concavity in Probabilistic Constrained Stochastic Programming. Operations Research Letters 39 (3), 188-192.

[30] Ruszczyński A. 2002. Probabilistic Programming with Discrete Distribution and Precedence Constrained Knapsack Polyhedra. Mathematical Programming 93, 195-215.

[31] Sarper H. 1993. Capital Rationing under Risk: A Chance-Constrained Approach Using Uniformly Distributed Cash Flows and Available Budgets. The Engineering Economist 39 (1), 49-76.

[32] Tanner M.W., Ntaimo L. 2010. IIS Branch-and-Cut for Joint Chance-Constrained Stochastic Programs and Application to Optimal Vaccine Allocation. European Journal of Operational Research 207, 290-296.

[33] van Ackooij W., Henrion R., Moller A., Zorgati R. 2011. On Joint Probabilistic Constraints with Gaussian Coefficient Matrix. Operations Research Letters 39, 99-102.

[34] van de Panne C., Popp W. 1963. Minimum-Cost Cattle Feed under Probabilistic Constraints. Management Science 9, 405-430. 